\title{
Optimal trading of imbalance options for power systems using an energy storage device
}

DOI:

10.1016/j.ejor.2018.09.037

\section{Document Version}

Accepted author manuscript

Link to publication record in Manchester Research Explorer

\section{Citation for published version (APA):}

Szabó, D. Z., Duck, P., \& Johnson, P. (2018). Optimal trading of imbalance options for power systems using an energy storage device. European Journal of Operational Research. https://doi.org/10.1016/j.ejor.2018.09.037

\section{Published in:}

European Journal of Operational Research

\section{Citing this paper}

Please note that where the full-text provided on Manchester Research Explorer is the Author Accepted Manuscript or Proof version this may differ from the final Published version. If citing, it is advised that you check and use the publisher's definitive version.

\section{General rights}

Copyright and moral rights for the publications made accessible in the Research Explorer are retained by the authors and/or other copyright owners and it is a condition of accessing publications that users recognise and abide by the legal requirements associated with these rights.

\section{Takedown policy}

If you believe that this document breaches copyright please refer to the University of Manchester's Takedown Procedures [http://man.ac.uk/04Y6Bo] or contact uml.scholarlycommunications@manchester.ac.uk providing relevant details, so we can investigate your claim.

\section{OPEN ACCESS}




\title{
Optimal trading of imbalance options for power systems using an energy storage device
}

\author{
Dávid Zoltán Szabó ${ }^{1, *}$, Peter Duck, Paul Johnson \\ School of Mathematics, University of Manchester, Oxford Road, Manchester M13 9PL, United Kingdom.
}

\footnotetext{
${ }^{*}$ Corresponding author

Email addresses: davidzoltan.szabo@postgrad.manchester.ac.uk (Dávid Zoltán Szabó), Peter.Duck@manchester.ac.uk
} (Peter Duck), paul.johnson-2@manchester.ac.uk (Paul Johnson) 


\title{
Optimal trading of imbalance options for power systems using an energy storage device
}

\author{
Dávid Zoltán Szabó ${ }^{1, *}$, Peter Duck, Paul Johnson \\ School of Mathematics, University of Manchester, Oxford Road, Manchester M13 9PL, United Kingdom.
}

\begin{abstract}
Energy storage devices are coming online in electricity markets around the world but are still considered an expensive solution to the load balancing problem. We propose a new market framework in which the owner of an electricity storage facility is able to optimally sell options and trade in the electricity market to add flexibility for the system operator at different times of the day. The storage operator has the possibility to optimally decide which option to offer, with the restriction of having the storage device in the appropriate mode and only at particular times. The system operator accepts these offers as possible long-term real-time balancing resorts. With the storage device in place, either electricity consumption or generated electricity can be increased in the network and in our framework this happens accordingly via the exercise of the corresponding option.

Using a stochastic model for the imbalance, we calculate the real value of the optimal trading strategy for the storage operator and at the same time we can calculate the balancing cost of this resort compared to a so called "target cost" for the system operator. These results reveal that with proper parameter choices, mutual benefit is available, i.e. a financial profit for the storage operator whilst the balancing cost can also be reduced for the electricity system operator. Our results are illustrated via numerical calculations which are carried out after having the parameters of the model fit to real UK data. Optimal operational strategies are also described using our analysis.
\end{abstract}

Keywords: OR in energy; Electric Energy Storage Systems; Optimal energy storage control policies; Real option; Power system balancing.

\section{Introduction}

In an ideal world the demand and supply sides of electricity are in equilibrium. In practice, however, there is an imbalance between actual generation and consumption; some possible reasons for this could be forecast errors or generator failures. These imbalances are therefore related to unpredictable events which are becoming more frequent as alternative energy sources are becoming more prevalent, since their intermittent nature implies a higher level of generation uncertainty [18, 6, 21, 25, 14. Hence the task of the system operator which is to ensure that actual energy generation and consumption balance is becoming more complicated. This difficulty is experienced in an increasing number of countries, however, in this work using historical data we focus on the UK. National Grid (NG) controls the high-voltage electric power transmission network in the UK that connects power stations and major substations and ensures that electricity generated anywhere in the country can be used to satisfy demand elsewhere. Thus, a major task of NG, which acts as the system operator, is to correct the actual imbalance via one of two actions depending on the sign of the actual imbalance:

\footnotetext{
${ }^{*}$ Corresponding author

Email addresses: davidzoltan.szabo@postgrad.manchester.ac.uk (Dávid Zoltán Szabó), Peter.Duck@manchester.ac.uk (Peter Duck), paul.johnson-2@manchester.ac.uk (Paul Johnson)
} 
- incremental actions, such as requesting additional generation or a reduction in demand; or support through financial incentives users to disconnect devices for a spell, when there is a shortfall in supply;

- decremental actions, such as requesting generation to be curtailed or demand increased, when there is an overproduction of energy.

The so called Balancing Services Incentive Scheme (BSIS) [3] is an overall balancing cost-minimisation incentive scheme for NG imposed by the regulator. This scheme involves target balancing costs that are then compared to the actual energy balancing costs of the system operator. According to BSIS, whatever NG over or under spends, compared to the target cost, is shared with the industry at a rate of $30 \%$, such that for every $£ 1$ of cost saved, the industry receives $£ 0.70$.

We work under the assumption that target balancing is associated with short-term balancing. Hence, the target balancing cost is related to the so called managed spot market (short-term) price. In the UK, the short-term market is known as the Balancing Mechanism 1, where offers are submitted shortly before real time. The BSIS states several possible ways to reduce energy balancing costs. One of these incentivises NG to enter into balancing services contracts with a counterparty. Furthermore, in general the system operator of a given country can negotiate long-term bilateral contracts with third-party balancing service providers in advance, to guard against possible concerns about the amount or the price of balancing resources that will be offered on the short-term basis (see page 60 of [19]). Allied with these and supported by [18, 6, 21, 25, 14, we propose an agreement between NG and a counterparty. This counterparty, namely the storage operator, is the owner of a battery that can physically store electricity.

\subsection{Literature review}

In order to better facilitate our work in the research area of optimal decisions on power systems, we provide here a short discussion of the current state of the literature. Our work primarily relates to the balancing of electricity load on the market, something which the uncertainty inherent in renewable energy sources makes it harder to model. [17] is one of many papers to review a variety of models used to forecast the electricity load that support decision makings in the electricity sector. Stochastic Differential Equations (SDEs) such as the one in this paper do not appear explicitly as they are not very reliable as forecasting tools. We know that the difficulty in forecasting also comes with a cost, 33 investigate the increasing ancillary costs for suppliers with a dynamic consumer pricing mechanism. They use a game-theoretic approach to establish that the current system of marginal cost pricing does not always achieve the socially optimal solution. In their system flexibility is added through customer interactions, whereas we propose the creation of a new options market. Making investment decisions in such a market can be difficult, and [7 consider a real options approach to value investment decisions of renewable energy sources by stochastically modelling electricity prices in a similar way to how we consider the energy storage device. The investment is considered from the perspective of a private investor, this paper also serves as a recommendation for market design.

Up to now we have mentioned papers relating to the energy market side of our work, but there are many papers that try to value storage facilities in a similar way to this paper. For an overview of the different types of storage and their costs, 22] outlines all of the current technologies that are available. They note that 'the benefits brought to power system operation by utilizing Electrical Energy Storage technologies need further exploration', and also that more economic analysis is required to establish how practical different solutions can be. By looking at the benefit for both the storage provider and the system operator, we hope to improve on this situation. If we were to focus just at the storage operator, all market participants have today the opportunity to rent a storage facility and use it to take advantage of the volatility in electricity price. When it comes to valuing a storage facility, the review paper [35] gives a comprehensive cost-benefit analysis of the investment in such a device. In order to do so they have to make simplifications about the market that the battery operates in and forecasting the profits is particularly difficult. This paper should complement this sort of analysis as it aims to give a more accurate estimation of the positive benefit of operating a storage facility to the whole market. Many other papers have also investigated the value of operating a storage facility (ignoring costs). In [20] the authors analyzed the value of a wind power-plant with an energy storage facility using a dynamic programming approach. They include some uncertainty with 
a forecast of wind supply but don't include any stochastic elements in their solution. Similar work in [10] investigated the optimal operation of a combined windhydro pumping storage power plant and discovered that considerable gains can be attained. Again a deterministic solution method is employed. Alternatively 8 take an approach similar to this paper, in which they consider a stochastic control problem to examine the valuation of energy storage facilities. They obtained probabilistic solutions based on optimal stopping problems which maximize the expected profit of the storage owner when buying and selling energy on the spot market. Also in a slightly different setting, 32 derived nonlinear partial-integro-differential equations to model the optimal operation of natural gas storage facilities. They also use similar techniques such as finite difference to solve the resulting problem. 34] consider a model where the storage is operated for profiteering reasons and the corresponding model involves several aspects such as utility functions, non-trivial storage capacity, etc. They have shown properties about the value of storage as a function of actual capacity. The main aim of each of these papers is strictly to maximize profits for the owner of the facility, even though it is also discussed in these papers that the increased number of storage devices can lead to mitigation of supply-demand fluctuation and so the network can also take benefit from these participants.

Several studies have investigated the social contributions of implementing energy storage facilities. [30] carried out a study on the UK by stating that a reasonable level of conventional sources still need to be retained in order to ensure energy security under conditions of high demand and low wind. The authors also discussed that energy storage facilities can mitigate the undesired effect of volatility imposed by renewable energy sources. 5] presented a model predictive control problem related to the operation of the storage and claimed that the economic costs of forecast uncertainties of renewable sources can be kept at an acceptable level with this approach. The effect on social welfare of the increased usage of electricity storage has been investigated in [28. The authors have found that electricity storage can smooth fluctuations between off peak and on peak periods. Furthermore a significant cost reduction can be achieved by deploying these storage units onto the grid which will lead to an improvement in social welfare. Incorporating dynamic aspects of demand and generation, [15] examined whether or not optimal storage operation matches socially optimal parameters under various ownership models of the storage unit such as supplier, consumer or stand-alone player. Apart from mitigating the volatility of electricity generation and electricity prices, they found that in cases when the storage facility is owned by the consumer or a stand-alone player, the optimal operation does not lead to maximising social welfare. In this paper, we also consider the notion of social welfare but from a slightly different point of view, that is by calculating the revenue for both system operator and storage operator of entering into our proposed agreement. We assume that the sum of the individual revenues represents the welfare contributed to society. Since the storage operator is a price-taker we assume that market price is not affected by the storage operation - which is realistic given the small size of the storage facility - thus the previously sketched out way seems appropriate to model the social benefits of the storage facility; also supported by the fact that there are no other parties involved into the agreement.

In this work the owner of the storage facility is a stand-alone player who provides options with energy as the underlying for sale to the network operator. We set optimization problems from the storage owner's point of view but also consider the objectives of the network operator which is to correct imbalances and so we rule out purely profiteering scenarios. Our research is motivated by the potential use of electricity storage to smooth fluctuations in supply and demand by using contracts that involve finite or infinite time horizon call or put options. A research problem for infinite time horizon call options has already been proposed in 24, where the system operator exercises the option according to a fixed threshold strategy to request additional energy from the storage operator when total demand exceeds generation. This study has been extended in 23. to allow for more general models of the instantaneous system imbalance and negative market prices, amongst other things. As an extension of [24, 31] analysed decremental balancing reserve service with put options using a similar stochastic basis, but modelled the price as a capped affine transformation of the imbalance. In this paper we propose an entirely new framework in which the storage operator is able to trade in both put and call options, as well as purchasing electricity on the market. By including a time dependent element to the problem, we must consider how and when options are traded to make this market work in the long term.

One task of this paper is to analyse the optimal operation of the battery among different available 
strategies in a setting that incentivises the owner of this battery to be engaged in offering options for the system operator. This means that we calculate the lifetime financial benefit of the optimal battery operation for the storage operator. Simultaneously, we compare the difference between the target balancing cost and the cost of this alternative strategy provided by this agreement for the system operator. Our main goal is to show that both parties can simultaneously achieve benefit by entering into such an agreement. This means that the sum of the two party's individual values is positive which can later be redistributed between the two parties. As discussed above we also consider this positive sum as a contribution to social welfare. Moreover, a complementary balancing tool is provided for the system operator by the options, which serves as a security against events when there is a failure on the provision of standard balancing actions.

We follow recommendations of 4 by applying the so called real options methodology that has become a successful tool in option pricing on electricity markets and has also been applied in [24, 31. According to this methodology, we take the discounted expectation of the different cash-flows under real world measure and so perform a real valuation of the optimal trading strategy for both parties. Henceforth, we refer to this corresponding problem value as the real value.

This paper is organised as follows. In Section 2, we establish a mathematical model for the imbalance volume and electricity price process using empirical data. In Section 3, we provide a detailed description of our model, relating the problem to the optimal switching model of [12]. In Section 4 we present results for our main cases, calculating the real value for both counterparties. We also show, via simulations, the optimal operational strategy of the storage operator for the end of the business day and for the start of the business day. This means that we present the optimal decisions of the storage operator against the imbalance horizon for different storage states. In Section 5 we consider the consequences of our work. In the appendices we provide supporting results for the numerical calculations.

\section{Methodology and empirical facts}

In this section we analyse empirical data in order to replicate a setting where we can facilitate a contract in an appropriate manner. We perform an analysis using historical UK imbalance volume and system price data from the Elexon portal [2] between September 2013 and April 2016. We are interested in stochastically modelling these two aforementioned quantities with a view to establishing a relationship between them. We seek to develop a model where all available market information is expressed accurately in these variables.

There are 48 settlement periods each day in the UK short-term imbalance market, meaning that every half an hour, a new imbalance volume and system price is calculated. In Figure 1 we can see how the imbalance volume changes on two different time scales covering an arbitrarily selected day and month. We can see that this process is very volatile, but appears to revert back to the zero level; mean reverting features are common in energy markets 9 . Thus, we choose an Ornstein-Uhlenbeck process to model the empirical imbalance volumes in the contract. This choice differs from [24, 31, 11] who used Brownian motions which have independent increments and can wander off to very large positive or negative values at longer-time scales. These previous studies, however, permit analytical results with this simpler assumption. The aim of this work is to provide numerical contract values over longer time periods, therefore we must choose this more realistic model for the imbalance volume. We can invoke [23] who already used mean-reverting feature to model imbalance price also supported by the statistical work of [27, 16].

Definition 1. The Ornstein-Uhlenbeck process is a stochastic process that satisfies the following stochastic differential equation:

$$
d X^{x_{0}}(t)=\kappa\left(\mu-X^{x_{0}}(t)\right) d t+\sigma d W(t), \quad X^{x_{0}}(0)=x_{0}
$$

where $\kappa>0, \mu$ and $\sigma>0$ are parameters, $W(t)$ denotes the Wiener process and $x_{0}$ is the starting point of the process $X^{x_{0}}$ at time $t=0$.

We used a Hessian matrix-based Maximum Likelihood Estimation method in R to estimate the parameters of the Ornstein-Uhlenbeck process for our empirical data points. Using the imbalance volume data points 


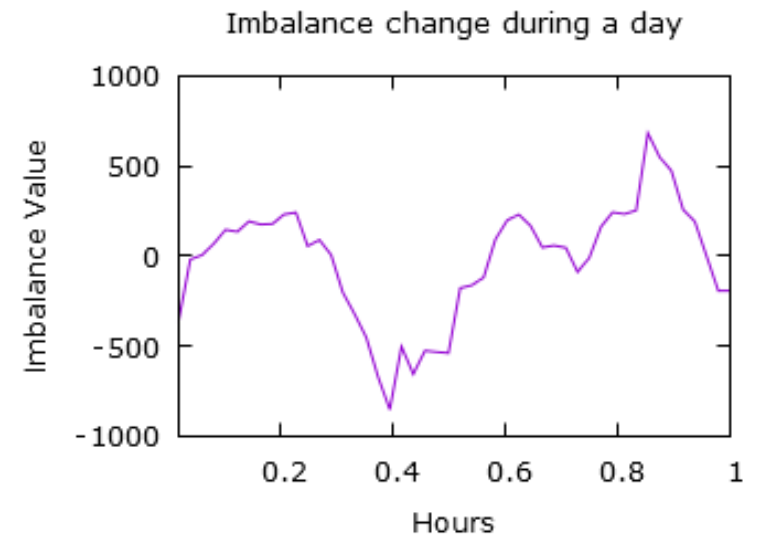

(a)
Imbalance change during a month

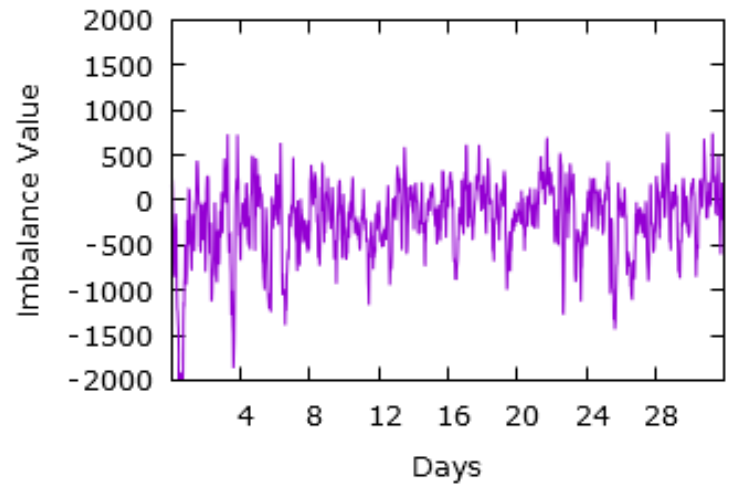

(b)

Figure 1: Imbalance volumes covering 1 day in subfigure (a) and 1 month in subfigure (b).

in the previously stated time range, the estimated parameters are: $\kappa=2310.1807, \sigma=24364.8525, \mu=$ -94.7489. Note that the unit of time is year, so these parameters are understood at a yearly level $(d t=1$ year) and had to be rescaled appropriately for the numerical calculations of our daily model.

Throughout our numerical calculations we will use the above model parameters with a small adjustment, the mean of the process $(\mu)$ will be assumed to be zero. This choice obviously simplifies our calculations but it is important to note that the value above is relatively small compared to the range where the process evolves $[-2000,2000]$. In this work we analyse both decremental and incremental balancing services by offering either put or call options. Our aim is to provide a symmetrical contract, meaning that the exercise decision for the two types of options will have the same absolute magnitude, and a non-zero choice of $\mu$ would complicate this matter. Furthermore, the aim of the system operator is to undertake balancing actions that ultimately force the imbalance back to zero; therefore the assumption that $\mu=0$ is also in line with this aim. We provide an analysis regarding the accuracy of this choice in Appendix B.

Now, we analyse the system price values. We consider these values as the price on the managed spot (short-term) market. Further, we assume that there exists a different liquid market, where the storage operator can buy (sell) electricity to charge (discharge) the battery without liquidity constraints. This is important, since the physical constraints can be only satisfied if the system operator is allowed to buy and sell electricity on this liquid market. We work under the assumption that these two (short-term, liquid) markets have mainly mutual participants and so the spot prices on these markets are equal. Henceforth we refer to this equal price as the 'electricity price'.

A detailed analysis about the electricity price is available in Appendix A, but for now we state its conclusion that is the electricity price is assumed to be given in the following form:

$$
f(t, x)=c(t)+b(t) x
$$

where $x$ is the current imbalance volume at $t \quad(0 \leq t \leq 1)$ which is the time of the business day and $c(t), b(t)$ represent the time dependent slope and intercept coefficients, respectively. This means that we have a linear dependence for each time point of the business day, however the slope and intercept parameters might change over the day to achieve a more accurate model. We have to note that the $R^{2}$ value of the linear regression increases from 0.2 by the constant linear dependence case to around 0.4 by the regressions of the time-dependent case, indicating that the imbalance model is more flexible and accurate when adding time dependent feature. 


\section{Lifetime contract valuation as an optimal switching problem}

This paper values a lifetime contract in which the system operator is obliged to buy put or call options from the storage operator for a fixed price during a given period in the day. We use a daily periodic model, meaning that the American-style options have a fixed end of the business day expiration date and the cycle starts again on a new business day. As an important contribution, this paper considers a simultaneous calculation of the real value for both parties and shows that a mutual benefit for both parties is possible. This happens via identifying the optimal decision for the storage operator.

\subsection{Preliminary description}

As a simplification, in our model there are two mutually exclusive operational states for the battery empty (0) and full (1). This means that without any transient states the battery is either empty or full and the charge-discharge activity happens in a single impulse. We assume that there exists an electricity market where participants can buy and sell electricity without liquidity issues. The storage operator is engaged in a finite number of market activities which depend on the battery's operational state:

\section{Battery Full}

(a) do nothing

(b) sell all of the electricity to the market

(c) i. sell a call option to the system operator

ii. sell a unit of electricity when the option is exercised

\section{Battery Empty}

(a) do nothing

(b) buy a unit of the electricity from the market

(c) i. sell a put option to the system operator

ii. buy a unit of electricity when the option is exercised

Note that once a call option has been sold, the battery can only reach the 'empty' state from 'full' if the system operator has exercised the option. Similarly, once a put option has been sold, the battery can only reach the 'full' state from 'empty' if the system operator has exercised the option. That is, no market activities are allowed for the storage operator if the system operator still owns any non-expired options to ensure that the storage operator meets the requirements of the option when it is exercised. It is also important to note that the battery has to be in the right state when an option is offered, meaning that a put option can only be sold when the battery is empty and a call option can only be sold when the battery is full. We will henceforth refer to a non-expired option as an 'Active Option'. Both option-related activities therefore contain two steps. The first step, c(i) is an insurance for the system operator which is given in return for the paid option premium. The storage operator thus ensures that they are able to buy or sell a unit of electricity if the system operator decides to smooth fluctuations by exercising the option, which is when the second step c(ii) occurs.

The battery's operational states and storage operator's market status can be combined in six different ways. These storage operator states are tabulated below, along with an indication of their validity.

\begin{tabular}{c|c|c|c|} 
& Active Call Option & Active Put Option & No Active Options \\
\hline Battery Full & Valid & Invalid & Valid \\
\hline Battery Empty & Invalid & Valid & Valid \\
\hline
\end{tabular}

We have now identified four valid storage operator states for the storage operator's activities:

1. the battery is currently full and no option has been sold

2. the battery is currently empty and no option has been sold 
3. the battery is currently full and a call option has been sold

4. the battery is currently empty and a put option has been sold

We define important time points during each business day related to when the parties become entitled to trigger different actions. In our setting, the storage operator can sell options to the system operator from the beginning of the business day $(t=0)$ until $t_{1}$. The system operator can then exercise these options from $t_{2}\left(t_{1}<t_{2}\right)$ until $T$, where $T$ denotes the end of the business day.

These time points are chosen to reflect the main objective of this work. The beginning of a business day is set to be the start of the period when options are sold to provide an alternative balancing opportunity for the system operator, that indeed covers a longer time range up to 24 hours than the short-term basis where offers are submitted shortly before real time. Furthermore, there may be a time gap $t_{2}-t_{1}$ when no option related activity happens. This gap plays an important role in our model since we assume that the system operator's exercise strategy is known a priori by the storage operator. Therefore, the gap yields some uncertainty about the future imbalance values and so it protects the system operator from those decisions of the storage operator that are clearly detrimental to the system operator and only make pure financial benefit for the storage operator, without providing real balancing services.

\subsection{An impulse control model}

Here we explicitly state our problem as an impulse control model following [12. To do this we first define the possible switches between the states. We denote the combined state space for the storage operator by $E=\{1,2,3,4\} \times \mathbb{R}^{+} \times \mathbb{R}$, which represents the actual storage operator state, the time point and the system imbalance. We also assume that $\mathcal{A}(i, t, x) \subseteq E$ denotes the set of possible combined states which are accessible from each given storage operator state at each time point under a given physical value. In our case this mapping is defined by

$$
\begin{aligned}
& \mathcal{A}(i, t, x)=\left\{\begin{array}{lll}
\{(1, t, x),(2, t, x),(3, t, x)\}, & \text { if } i=1 \\
\{(2, t, x),(1, t, x),(4, t, x)\}, & \text { if } i=2 \\
\{(3, t, x)\}, & \text { if } i=3 \\
\{(4, t, x)\}, & \text { if } i=4
\end{array} \quad \text { for } \quad 0<t \leq t_{1}\right. \\
& \mathcal{A}(i, t, x)=\left\{\begin{array}{ll}
\{(1, t, x),(2, t, x)\}, & \text { if } i=1 \\
\{(2, t, x),(1, t, x)\}, & \text { if } i=2 \\
\{(3, t, x)\}, & \text { if } i=3 \\
\{(4, t, x)\}, & \text { if } i=4
\end{array} \quad \text { for } \quad t_{1}<t \leq t_{2}\right. \\
& \mathcal{A}(i, t, x)=\left\{\begin{array}{lr}
\{(1, t, x),(2, t, x)\}, & \text { if } i=1 \\
\{(2, t, x),(1, t, x)\}, & \text { if } i=2 \\
\{(3, t, x)\}, & \text { if } i=3 \text { and } x>x_{c}^{*} \\
\{(2, t, x)\}, & \text { if } i=3 \text { and } x \leq x_{c}^{*} \\
\{(4, t, x)\}, & \text { if } i=4 \text { and } x<x_{p}^{*} \\
\{(1, t, x)\}, & \text { if } i=4 \text { and } x \geq x_{p}^{*}
\end{array} \quad \text { for } \quad t_{2}<t<T\right. \\
& \mathcal{A}(i, t, x)=\left\{\begin{array}{ll}
\{(1, t, x)\}, & \text { if } i=1 \\
\{(2, t, x)\}, & \text { if } i=2 \\
\{(1, t, x)\}, & \text { if } i=3 \\
\{(2, t, x)\}, & \text { if } i=4
\end{array} \quad \text { for } \quad t=T\right.
\end{aligned}
$$

We refer to $\mathcal{A}(i, t, x)$ as the set of admissible impulses given $(i, t, x)$. The rationale behind the mappings in (3)-6 is as follows. As we have already stated, we split each business day into different parts, and we 
allow different actions for the storage operator depending on the current part. Option (put and call) selling is only allowed from time $t=0$ to $t=t_{1}$, and exercising the option is only allowed from $t=t_{2}\left(\geq t_{1}\right)$ to $t=T$, where $T$ denotes the end of the business day when non-exercised active options expire. It is important to emphasize that selling the option is the decision of the storage operator, whereas the exercise of the option happens according to a fixed strategy by the system operator, who we assume is always willing to buy the option. That is, whenever the physical imbalance $X^{x_{0}}(t)$ exceeds a fixed threshold $x_{p}^{*}$ for the first time after $t=t_{2}$, an active put option is exercised. Similarly, whenever the physical imbalance $X^{x_{0}}(t)$ goes under a fixed threshold $x_{c}^{*}$ for the first time after $t_{2}$, an active call option is exercised. It is important to note that this decision strategy is available for the storage operator. Furthermore, we only allow the storage operator to have one active option at any time, due to the physical constraints of the battery. We also let the storage operator sell and buy electricity on the electricity market if there are no active options, without any liquidity issues. However, we would also like to replicate the effect of degradation in the storage capacity of the storage device, by including a constant $C_{2}$ maintenance cost that happens at each charge or discharge activity. This maintenance cost is a burden for the storage operator, meaning that we do not use a model where only a fraction of energy is considered by the charge/discharge actions or in different words where each charge-discharge cycle reduces the capacity by a factor $A \in(0,1)$, instead perfect charge/discharge is available for the storage operator but only with a significant cost. Moreover, we also use a sufficiently large transaction cost $C_{1}>0$ for each single trading on the electricity market. This cost includes the previously mentioned $C_{2}$ maintenance cost associated to the charge/discharge activities, furthermore we assume that a $C_{1}-C_{2}$ bid-ask spread is present in this market. This $C_{1}-C_{2}$ bid-ask spread is large enough in our model to disincentivise the storage operator from procuring any substantial financial gain by excessively trading on the electricity market and to truly set the focus on providing a balancing service for the system operator by engaging in selling the options. This spread is constant during the day to simplify our model, and its exact value is chosen in our calculations to match the magnitude of the daily buy-sell spread of electricity price $f$. The values of these costs can be seen in Table 1. $C_{2}$ is chosen to be 5 by default, meaning that its value is around $10 \%$ of the average electricity price of the market. This maintenance cost is aimed to cover the cost to both run and maintain the battery in good operating shape. We are confident that although this cost is significant, it is not exceedingly large.

If the system operator does not exercise an active option, implying that it expires at $t=T$, then the battery remains in the same state and obviously there is no effect on the system imbalance volume. If the system operator exercises a put option, then they will receive one unit of electricity from the system operator as soon as the imbalance volume process enters the set $\left[x_{p}^{*},+\infty\right)$. It is assumed that this exchange has negligible impact on the system imbalance volume at that point. Likewise, if the system operator exercises a call option, then they will sell one unit of electricity to the system operator as soon as the imbalance volume process enters the set $\left(-\infty, x_{c}^{*}\right]$. It is again assumed that this exchange has negligible impact on the system imbalance volume at that point.

We are interested in the optimal timing decision for the storage operator under the aforementioned conditions, by calculating the lifetime real value with the stated daily periodicity. To do so we first define the associated cash flows linked to the different storage operator state switches for both parties and then the optimal switching problem itself.

\subsubsection{Storage operator state changes and associated cash flows}

Let us consider the possible cash flows for the storage operator, with $(x, t) \in \mathbb{R} \times \mathbb{R}^{+}$denoting the current imbalance level at a given time point:

(a) In storage operator state 1 the storage operator receives $f(x, t)-C_{1}$ for selling a unit of electricity on the electricity market and moves to storage operator state 2 .

(b) In storage operator state 1 the storage operator receives $p_{c}$ for selling a call option to the system operator and moves to storage operator state 3 .

(c) In storage operator state 2 the storage operator pays $f(x, t)+C_{1}$ for buying a unit of electricity on the electricity market and moves to storage operator state 1 . 
(d) In storage operator state 2 the storage operator sells the put option to the system operator for a premium $p_{p}$ and moves to storage operator state 4 .

(e) In storage operator state 3 the storage operator waits until the system operator exercises the call option and pays the strike price $K_{c}$ to the storage operator and also pays a $C_{2}$ maintenance cost, after which a transition to storage operator state 2 happens. If this does not happen before $t=T$, the storage operator moves to storage operator state 1 at this time $t=T$ without having any cash flow.

(f) In storage operator state 4 the storage operator waits until the system operator exercises the put option and receives the strike price $K_{p}$ from the storage operator and pays a $C_{2}$ maintenance cost, after which a transition to storage operator state 1 happens. If this does not happen before $t=T$, the storage operator moves to storage operator state 2 at this time $t=T$ without having any cash flow.

These are the possible cash-flows between the two parties, which we use to develop a model for the cost of impulse control, which is a mapping

$$
((i, t, x),(j, t, y)) \mapsto K((i, t, x),(j, t, y)): E \times E \rightarrow \mathbb{R}
$$

defined only for $(j, t, y) \in \mathcal{A}(i, t, x)$. As we can see by the possible values of $\mathcal{A}(i, t, x)$ above, we can neglect the trivial time and imbalance $(t, x)$ coordinates and write the cost as a matrix of the different storage operator states. The row of the matrix represents the state from which the transition happens and the column to which the transition is made. This means that entry $(i, j)$ of the matrix indicates the cost of the transition from storage operator state $i$ to storage operator state $j$.

$$
K(i, j)=\left(\begin{array}{cccc}
0 & -f(t, x)+C_{1} & -p_{c} & I . P . \\
f(t, x)+C_{1} & 0 & I . P . & -p_{p} \\
0 & -K_{c}+C_{2} & 0 & I . P . \\
K_{p}+C_{2} & 0 & I . P . & 0
\end{array}\right)
$$

The entry I.P. denotes that the storage operator state change is impossible, and therefore has no associated cost.

\subsubsection{Associated cash flows of the system operator}

The system operator is constantly required to undertake balancing actions in order to meet the energy demand with sufficient supply. In particular in the UK, the target scheme (BSIS) [3] contains regulations on how the NG is responsible for performing these tasks and so to pay the balancing costs. In this paper we compare the target balancing cost with the actual outcome costs of this model. This target balancing cost is calculated as the product of the current imbalance volume and the electricity price. If there is an energy shortfall, then this imposes a cost for the system operator. That is, the system operator is required to buy the unit of electricity and to pay the price on the short-term market after each unit of shortage. In an analogous way, in case there is an energy surplus with insufficient demand from consumers, then the extra electricity will also impose a balancing action on the system operator. The system operator has to sell the extra amount of electricity on the short-term market. During these selling transactions they receive the electricity price on the market.

We will compare the balancing cost provided by the agreement to this target short-term balancing cost. The following matrix shows how the cost of the system operator $K_{N . G}$. changes by the different impulses relative to the target. Here, following the cost matrix to the storage operator we disregard the time and imbalance $(t, x)$ coordinates and write the cost as a matrix of the different storage operator states. As with (7), the row of the matrix represents the state from which the transition happens and the column the state to which the transition is made. This means that entry $(i, j)$ of the matrix indicates the cost of the transition 
from storage operator state $i$ to storage operator state $j$.

$$
K_{N . G .}(i, j)=\left(\begin{array}{cccc}
0 & 0 & p_{c} & I . P . \\
0 & 0 & I . P . & p_{p} \\
0 & K_{c}-f(t, x) & 0 & I . P . \\
-K_{p}+f(t, x) & 0 & I . P . & 0
\end{array}\right)
$$

Again, the entry I.P. denotes that the storage operator state change is impossible, and therefore has no associated cost.

As the holder of the option, the system operator has a cost $p_{p}\left(p_{c}\right)$ whenever he buys the put (call) option. Further, when an option is exercised, depending on the type, the system operator receives $K_{p}$ or pays $K_{c}$. At these time points a balancing correction also happens, since this is why the system operator deals with options. Therefore, we need to compare this balancing cost with the previously stated target. As a consequence, the change in the balancing cost is $K_{c}-f(t, x)$ for a call and $-K_{p}+f(t, x)$ for a put option at the times when the options are exercised.

In our numerical scheme, we calculate the cost change for the system operator in parallel with the financial gain of the storage operator, by assuming that optimal decisions are made by the storage operator.

\subsubsection{Relation to variational inequalities}

We now formulate our problem and verify that it is a solution of a system of variational inequalities. Mathematically speaking, the imbalance volume process $X^{x_{0}}(t)$ is defined on a filtered probability space $\left(\Omega, \mathcal{F},\left(\mathcal{F}_{t}\right)_{t \geq 0}, \mathrm{P}_{x}\right)$. As we have stated, we model this imbalance volume process as an Ornstein-Uhlenbeck process independently of the actual storage operator state $i(=1,2,3,4)$. We use a joint process $\left(t, X^{x_{0}}(t)\right) \in$ $\hat{E}$, where $\hat{E}=\mathbb{R}^{+} \times \mathbb{R}$ to model the time dependent feature of our model. Finally, we consider the space $E=\{1,2,3,4\} \times \hat{E}$ and the stochastic process $Y$ with uncontrolled distribution $\left(i, t, X^{x_{0}}(t)\right)$, when started in $(i, t, x) \in i \times \hat{E}$.

We define the so called controlled measure $\left(\mathbb{P}_{z}^{S}\right)_{z \in E}$ in the same manner as in [12]. That is, under this measure between each two random times $\tau_{n-1}<\tau_{n}$, the process $Y$ runs uncontrolled with the previously defined uncontrolled distribution $\left(i, t, X^{x_{0}}(t)\right)$. The random times $\tau_{n}$ are associated with the possible state switches meaning that at each random time $\tau_{n}$ an impulse is exercised and the process is restarted at the new combined state space $\gamma_{n} \in E$. We associate our strategy with impulse control strategies, which are sequences $S=\left(\tau_{n}, \gamma_{n}\right)_{n \in \mathbb{N}}$. By using the cost functional $K$ defined in the previous subsection we can define the optimal switching problem for the storage operator, following the notation of [12]:

$$
v(z)=\sup _{S=\left(\tau_{n}, \gamma_{n}\right)_{n}} \mathbb{E}_{z}^{S}\left\{-\sum_{n=0}^{\infty} e^{-r \tau_{n}} K\left(Y\left(\tau_{n},-\right), Y\left(\tau_{n}\right)\right)\right\}, \quad z \in E
$$

among all admissible impulse control strategies, that are: $S=\left(\tau_{n}, \gamma_{n}\right)_{n \in \mathbb{N}}, \tau_{n} \rightarrow \infty$ as $n \rightarrow \infty$. This $v(z)$ is the real value of the storage operator for a given $z \in E$. This means that the storage operator chooses the strategy among the available ones which leads to maximising their discounted expected cash-flow reflecting the objective to optimally operate the battery. We also formulate the real value of the system operator. According to the agreement the system operator willingly accepts the offered options, therefore the real value is determined by the optimal decision of the storage operator. Denoting by $\hat{S}=\left(\hat{\tau}_{n}, \hat{\gamma}_{n}\right)_{n \in \mathbb{N}}$ the supremum of the admissible impulse control strategies, that is the optimal strategy of the storage operator, we can formulate the real value $(w(\cdot))$ of the system operator for a given $z \in E$ :

$$
w(z)=\mathbb{E}_{z}^{\hat{S}}\left\{-\sum_{n=0}^{\infty} e^{-r \hat{\tau}_{n}} K_{N . G .}\left(Y\left(\hat{\tau}_{n},-\right), Y\left(\hat{\tau}_{n}\right)\right)\right\}, \quad z \in E .
$$

We must also satisfy a condition on the negative section of the cost function $\left(K^{-}\right)$for all admissible impulse control strategies, by applying the results of [12, namely:

$$
\mathbb{E}_{z}^{S}\left\{\sum_{n=0}^{\infty} e^{-r \tau_{n}} K^{-}\left(Y\left(\tau_{n},-\right), Y\left(\tau_{n}\right)\right)\right\}<\infty, \quad z \in E .
$$


The financial interpretation of (9) can be understood in a simple way. The storage operator optimally operates the battery by optimally choosing from the available possibilities, and this choice is reflected by taking the supremum from the stopping times that corresponds to the state switches that are possible for the storage operator. The condition (11) on the negative side of the cost function $K$ clearly needs to be satisfied in order to avoid an infinite financial profit for the storage operator and to make the problem realistic. The discount factors $\left(e^{-r \tau_{n}}\right)$ between stopping times are crucial and play important roles in order to get a financially meaningful problem.

Using the time-homogeneous, Markovian nature of the underlying process, we can apply the findings of 12]. To do this, we introduce $r$-excessive ( $r$-superharmonic) functions [13] (see also [26]):

Definition 2. A measurable function $\Phi: E \rightarrow[0, \infty]$ is said to be $r$-excessive (relative to $X$ ) if:

1. $\Phi$ is r-superaveraging:

$$
e^{-r t} \mathbb{E}_{x}\{\Phi(X(t))\} \leq \Phi(x), \quad \forall \quad x \in E \text { and } t \geq 0 .
$$

2. $\lim _{t \downarrow 0} e^{-r t} \mathbb{E}_{x}\{\Phi(X(t))\}=\Phi(x), \quad \forall \quad x \in E$.

Following the arguments of [12] we define a set:

$$
H:=\{h: E \mapsto \mathbb{R} \mid \quad h \quad \text { is } \quad r \text {-excessive, } \quad h \geq 0, \quad h \geq \mathcal{M}(h)\}
$$

where the maximum operator $\mathcal{M}$ is given by:

$$
\mathcal{M} \omega(x)=\sup _{y \in \mathcal{A}(x) \backslash\{x\}}\{\omega(y)-K(x, y)\} .
$$

We assume that there indeed exists an optimal impulse control strategy $\hat{S}=\left(\hat{\tau}_{n}, \hat{\gamma}_{n}\right)$ for the problem defined in (9). Then by corollary 2.4 of [12], $v$ is the pointwise minimiser of the set $H$. Using the assumption that the solution for the problem is smooth, which is based on the smoothness of the time-dependent price stack function, we can guarantee that the infinitesimal (Dynkin) $\mathbb{L}$ operator exists to express the value function as a solution of the related quasi-variational inequality. We provide the following lemma for confirmation.

Lemma 3.1. If a function $v: E \mapsto \mathbb{R}$ is r-excessive, bounded, and its second partial derivatives exist, then $(\mathbb{L}-r) v(x) \leq 0$.

Proof We know the following by $r$-superaveraging property:

$$
\frac{\mathbb{E}_{x}\{v(X(t))\}-v(x)}{t}=\frac{e^{r t} \mathbb{E}_{x}\left\{e^{-r t} v(X(t))\right\}-v(x)}{t} \leq \frac{e^{r t} v(x)-v(x)}{t}=\frac{v(x)\left(e^{r t}-1\right)}{t}
$$

then taking the limit of both sides yields:

$$
\mathbb{L} v(x) \leq \lim _{t \rightarrow 0} \frac{v(x)\left(e^{r t}-1\right)}{t}=\lim _{t \rightarrow 0} r v(x) e^{r t}=r v(x),
$$

where the first equality is derived by applying L'Hopital's rule.

We also know from [12] that the solution for $v$ is r-harmonic in the continuation region, implying that $(\mathbb{L}-r) v(z)=0$. Then, we claim that the value function is the solution of the following system:

$$
\max \{(\mathbb{L}-r) v(z), \mathcal{M} v(z)-v(z)\}=0, \quad \text { for all } \quad z \in E
$$

by also noting that $v(z)$ is $r$ - harmonic on the continuation region $(\mathcal{M} v(z)-v(z)<0)$, that is $(\mathbb{L}-r) v(z)=0$. Further, the optimal switching is associated with the $\mathcal{M} v(z)-v(z)=0$ set.

We solve this system of equations with a finite-difference scheme recursively against the number of days. We then compare the results received for different numbers of days for a given $z \in E$ and check if we have achieved a periodic state. If the change is insignificant, we stop the computation. We verify by our numerical calculations that with the help of a constant $\operatorname{cost} C$ on the electricity market the bounded condition (11) holds. 
Remark 3.2. As also discussed in [23] mean reverting processes are not amenable to analytical treatment as the infinitesimal generator of these processes is relatively complex. They obtained solutions for their problems which are explicit, on the other hand they still not available in analytical forms. As our problem is made more complex by the inclusion of finite time horizon options and therefore more realistic we are restricted to a numerical treatment.

\section{Results}

Here we present results to the problem described in the previous section. These results are the numerical solutions of the corresponding system of variational inequalities (17). As described previously, the agreement between the two parties has a daily feature, having an end of business day expiration date for both the offered call and put options. Therefore, we calculate the lifetime value of the problem to achieve a periodic state, in order to attain a stable solution (independent of initial conditions, once transients have decayed sufficiently). Over shorter times the contract value may depend on the initial state, but after reaching the periodic state this dependence is greatly reduced allowing us to offer representative values of the system. Given an NPV over an infinite time frame, annuities can be calculated by multiplying the NPV by the interest rate. That means that if we consider an agreement between the two parties such that they will enter into the contract for a given time frame, our total NPV values over the infinite horizons can be used to indicate the value of the contract over these shorter time frames. If the results are positive in the infinite time horizon case, then it can be concluded that we expect them to be positive over a shorter number of years. This obviously depends on the interest rate; throughout our calculations as a reasonable choice we use $r=0.1$ per annum.

We distinguish here two main cases depending on the realization of $t_{1}$ and $t_{2}$. In Case (i) we set $0<t_{1}=t_{2}$, whereas in the Case (ii) $0=t_{1}<t_{2}$. In Case (i) the storage operator is allowed to sell options to the system operator from the business day beginning $(t=0)$ until time $t_{1}$. From this $t_{1}=t_{2}$ time point until the end of the business day $(t=T)$ the system operator can exercise the active option. In Case (ii) the storage operator can only offer option at the beginning of the business day, that is $t_{1}=0$. There is a time lag between this time point and the first time point when the option can be exercised by the system operator, namely $t_{2}$.

We will conclude that Case (ii) has better properties over Case (i) meaning that options are more often exercised and so a more effective balancing opportunity is provided. Further, this Case (ii) has more favorable properties, that is to provide long-term balancing service for the system operator. This is simply due to the fact that there is a longer time to possibly exercise the option and there is also a fixed minimum time difference between the option offer date and its first possible exercise date $\left(t_{2}-t_{1}\right)$.

Hence, a more detailed and accurate analysis is provided for the aforementioned Case (ii). We perform our analysis with reasonable parameter assumptions that are in association with two sustainability conditions that are crucial from the system operator's point of view to enter into the daily agreement with the storage operator.

$$
\begin{aligned}
& \forall \quad t \in\left[t_{2}, T\right]: 0<p_{p} \leq K_{p}-f\left(x_{p}^{*}, t\right) \\
& \forall \quad t \in\left[t_{2}, T\right]: 0<p_{c}+K_{c} \leq f\left(x_{c}^{*}, t\right) .
\end{aligned}
$$

The explanations behind these conditions are as follows. We work under the assumption that the offered call and put options are exercised when the imbalance value hits certain threshold levels for the first time in the exercise region. The system operator can still perform balancing duties by selling the surplus of electricity or buying the shortage of electricity on the short-term market. In case 18$)$ and $(19)$ do not hold then there is no added benefit for the system operator, since this option-related balancing alternative is not cheaper than trading on the short-term market.

This means that if there exists a $t \in\left[t_{2}, T\right]$ such that $f\left(x_{p}^{*}, t\right)>K_{p}-p_{p}$ then the system operator can buy electricity cheaper on the short-term market than the aggregated payoff they face by using options, in case the exercise of the option happens at this time point $t$. Likewise, if there exists a $t \in\left[t_{2}, T\right]$ such that 


\begin{tabular}{|c|c|c|c|c|c|c|c|c|c|c|}
\hline Number & $p_{p}$ & $p_{c}$ & $K_{p}$ & $K_{c}$ & $x_{p}^{*}$ & $x_{c}^{*}$ & $\mathrm{r}$ & $C_{1}$ & $C_{2}$ if not mentioned & Violates? \\
\hline I. & 15 & 15 & 65 & 30 & 200 & -200 & 0.1 & 25 & 5 & NO \\
\hline II. & 5 & 5 & 65 & 30 & 200 & -200 & 0.1 & 25 & 5 & NO \\
\hline III. & 10 & 10 & 65 & 30 & 200 & -200 & 0.1 & 25 & 5 & NO \\
\hline IV. & 20 & 20 & 65 & 30 & 200 & -200 & 0.1 & 25 & 5 & NO \\
\hline V. & 25 & 25 & 65 & 30 & 200 & -200 & 0.1 & 25 & 5 & NO \\
\hline VI. & 15 & 15 & 60 & 35 & 200 & -200 & 0.1 & 25 & 5 & $(18)$ and 19 \\
\hline VII. & 15 & 15 & 62.5 & 32.5 & 200 & -200 & 0.1 & 25 & 5 & $(18)$ \\
\hline VIII. & 15 & 15 & 67.5 & 27.5 & 200 & -200 & 0.1 & 25 & 5 & NO \\
\hline IX. & 15 & 15 & 70 & 25 & 200 & -200 & 0.1 & 25 & 5 & NO \\
\hline X. & 15 & 15 & 65 & 30 & 100 & -100 & 0.1 & 25 & 5 & NO \\
\hline XI. & 15 & 15 & 65 & 30 & 300 & -300 & 0.1 & 25 & 5 & NO \\
\hline XII. & 15 & 15 & 65 & 30 & 400 & -400 & 0.1 & 25 & 5 & NO \\
\hline XIII. & 15 & 15 & 65 & 30 & 500 & -500 & 0.1 & 25 & 5 & \\
\hline
\end{tabular}

Table 1: Base setting combinations.

$p_{c}+K_{c}>f\left(x_{c}^{*}, t\right)$ then the system operator pays less money by buying the shortfall of electricity on the short-term market than the aggregated payoff they realise by using the options, in case the exercise of the option happens at this time point $t$.

Both of these cases are examples when the short-term way might be cheaper and so it is not unequivocally favourable for the system operator to use the options. Our assumption is that such cases are unacceptable for the system operator, and so we would like to rule out any of these possibilities. We provide (18) and (19) which imply that we can guarantee that the balancing opportunity by using options cannot be more expensive than short-term balancing at any time point in the exercise region $\left[t_{2}, T\right]$.

Our different parameter combinations will reflect these conditions and we will state for all our cases whether the sustainability conditions are satisfied or not.

We use 13 different parameter settings and these can be found in Table 1 (see also Appendix B for a detailed analysis). The table also reveals if (18) and (19) are satisfied or not. Throughout this work we use the parameter settings presented in this Table 1. We also apply a general setting with fixing the exercise region $\left[t_{2}, T\right]$, the one exception is when we analyse the real value for different exercise periods. Following a practical view, the standard choice for our analysis is a $22-2$ hour split of the business day, meaning that the second period when the system operator can exercise an active option lasts 2 hours. We also set the 'end of the business day' to be $6 \mathrm{pm}$ in our time-dependent work, meaning that $t_{2}=4 \mathrm{pm}$ and $T=6 \mathrm{pm}$. With this 4pm-6pm period choice we cover the busiest hours in the UK market when there is a peak in demand which can cause spikes in price and a problem for the system operator in short-term balancing.

\subsection{Numerical results for Case (i)}

In this subsection we analyse the contract results related to Case (i). Remember that the day is split into two parts, in this Case (i), and during the first part the storage operator is allowed to sell options, but they are not allowed to during the second part. Conversely, the system operator can only execute active options during the second part of the day.

Firstly, we wish to demonstrate that the values for both parties will converge to a periodic solution if we price the contract over a large number of days. We do this by plotting the real value at a particular point in space and time during the day, given that we apply a terminal condition of $v=0$ after the given number of days. In Figure 2 (for parameter combination I) and in Figure 3 (for parameter combination IV) we plot these real values, and we can see that if the terminal condition is applying after more that 15000 days there is virtually no change in value on this scale, therefore we can accept the result at 20000 days as a good approximation to the lifetime value using our fixed annual interest rate $r=0.1$. We have to note that the 
Real values for both parties

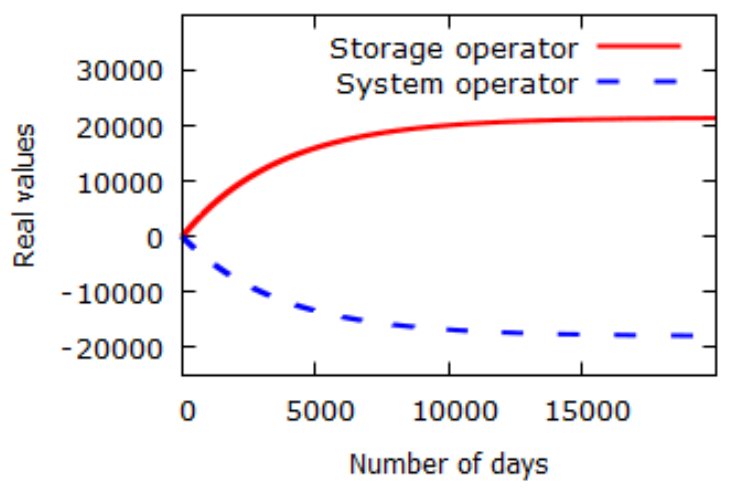

(a) Individual values
Real values for both parties

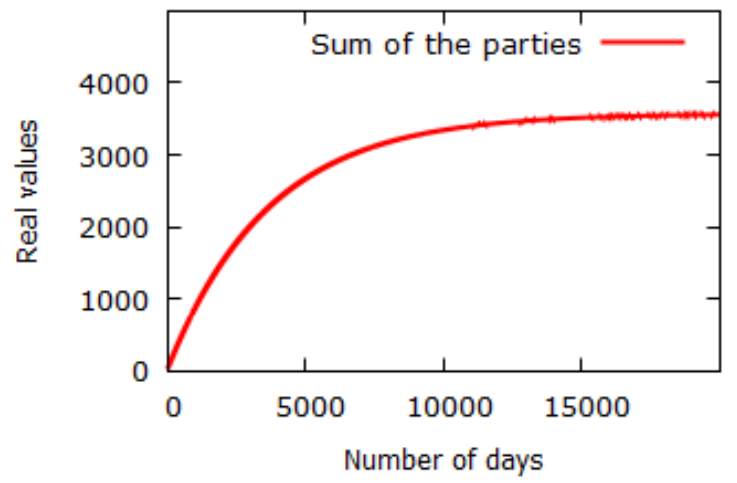

(b) Sum value

Figure 2: Corresponding real values to Case (i) under parameter combination I applying grid choice A against increasing numbers of days. The contract values denote the value in the beginning of the business day under 0 imbalance value and for initial case $(1, N)$. The second time interval is between $4 \mathrm{pm}$ and $6 \mathrm{pm}$, thus $T=6 \mathrm{pm}$ is the end of the business day.

calculation with 20000 days is solely carried out to offer a stable solution (independent of initial conditions and once transients have decayed sufficiently), and we do not propose an agreement between the two parties over 20000 days. We can see that the positive total profit is also observed on a shorter time frame, so the main contribution of this study about positive total profit can be concluded using more realistic time frame contracts.

In the next example of our analysis, we demonstrate the statistics of whether or not the option is sold and in particular at what time it is likely to happen. To do this we use Monte-Carlo simulation to create 5000 random trajectories of the underlying imbalance process, so given the optimal strategy of the storage operator we are able to infer what decisions they would make at a particular time. We find that Case (i) provides more flexibility for the storage operator when they make an optimal selling decision, due to the fact that there is a longer time period over which option selling is allowed. This becomes important later on, when determining what sort of market for selling these options can provide net benefit to both parties.

We state the frequency at which options are bought as a percentage of the 5000 total, and further the frequency at which an option is exercised during a day for a range of different parameter settings, initial states and imbalance values as given in Table 2. We can see that as the premium price increases that percentage of sold options also increases. This phenomenon reflects a very rational decision such that the storage operator is more likely to sell options for higher premium prices. This table also reveals that the difference of sold and exercised options is insignificant among the different starting imbalance values for same parameter combinations and initial states, but on average more options are sold from initial state $(0, \mathrm{~N})$ than from initial state $(1, N)$. This can be explained by the fact that the parameters we have set for the two options are not entirely symmetrical and so this can cause slightly asymmetric results. In Figure 4 we can see the frequency of the time at which options are sold during the day. We see that almost all options are sold very close to $t=t_{1}=t_{2}$. These simulations indicate that the storage operator's strategy is to wait as long as possible before selling the option so that they can be more certain as to whether the option will be exercised or not. This is a reasonable strategy, since a pure financial gain could be achieved by selling options that are unlikely to be exercised as the storage operator receives the option premium. Obviously allowing such a strategy in the market cannot provide value for the system operator who has to buy options that are never exercised. As a result we look instead to impose a regulatory framework where options can only be traded in advance, so that the market participants cannot be certain about whether they will be exercised or not. This leads us on to Case (ii), which aims to develop this framework and demonstrate that mutual benefit can be obtained. 


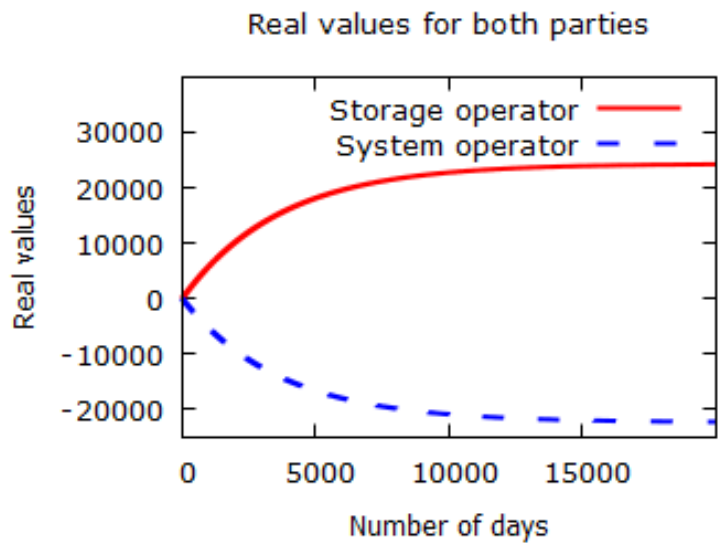

(a) Individual values

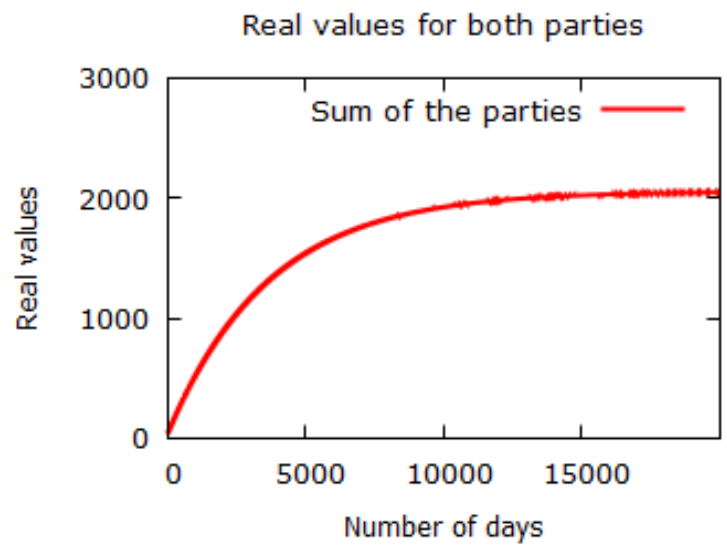

(b) Sum value

Figure 3: Corresponding real values to Case (i) under parameter combination IV applying grid choice A against increasing numbers of days. The contract values denote the value in the beginning of the business day under 0 imbalance value and for initial case $(1, N)$. The second time interval is between $4 \mathrm{pm}$ and $6 \mathrm{pm}$, thus $T=6 \mathrm{pm}$ is the end of the business day.

\begin{tabular}{lllll}
\hline & \multicolumn{1}{c}{ Initial imbalance $X(0)$} & $\mathbf{- 3 0 0}$ & $\mathbf{0}$ & \multicolumn{1}{c}{$\mathbf{3 0 0}$} \\
\hline \multirow{5}{*}{ Combination III } & Frequency of bought options initial state $(1, N)$ & $38 \%$ & $38 \%$ & $37 \%$ \\
& Frequency of exercised options initial state $(1, N)$ & $8 \%$ & $8 \%$ & $7 \%$ \\
& Frequency of bought options initial state $(0, N)$ & $42 \%$ & $43 \%$ & $42 \%$ \\
& Frequency of exercised options initial state $(0, N)$ & $9 \%$ & $10 \%$ & $9 \%$ \\
\hline \multirow{5}{*}{ Combination I } & Frequency of bought options initial state $(1, N)$ & $54 \%$ & $53 \%$ & $53 \%$ \\
& Frequency of exercised options initial state $(1, N)$ & $16 \%$ & $16 \%$ & $16 \%$ \\
& Frequency of bought options initial state $(0, N)$ & $53 \%$ & $53 \%$ & $53 \%$ \\
& Frequency of exercised options initial state $(0, N)$ & $15 \%$ & $16 \%$ & $16 \%$ \\
\hline \multirow{5}{*}{ Combination IV } & Frequency of bought options initial state $(1, N)$ & $65 \%$ & $63 \%$ & $63 \%$ \\
& Frequency of exercised options initial state $(1, N)$ & $25 \%$ & $23 \%$ & $24 \%$ \\
& Frequency of bought options initial state $(0, N)$ & $63 \%$ & $65 \%$ & $64 \%$ \\
& Frequency of exercised options initial state $(0, N)$ & $23 \%$ & $24 \%$ & $24 \%$ \\
\hline
\end{tabular}

Table 2: A table containing the results of the simulations. Using these simulations we calculated the frequencies at which options are sold and exercised on a given day under Case (i) for different parameter combinations and initial parameters applying grid choice A. The second time interval is between $4 \mathrm{pm}$ and $6 \mathrm{pm}$, thus $T=6 \mathrm{pm}$ is the end of the business day. Repetition of days is performed up to 20000 and the sample size is 5000 for each calculation. The frequencies are rounded to the nearest integer. 
Frequency of sold options

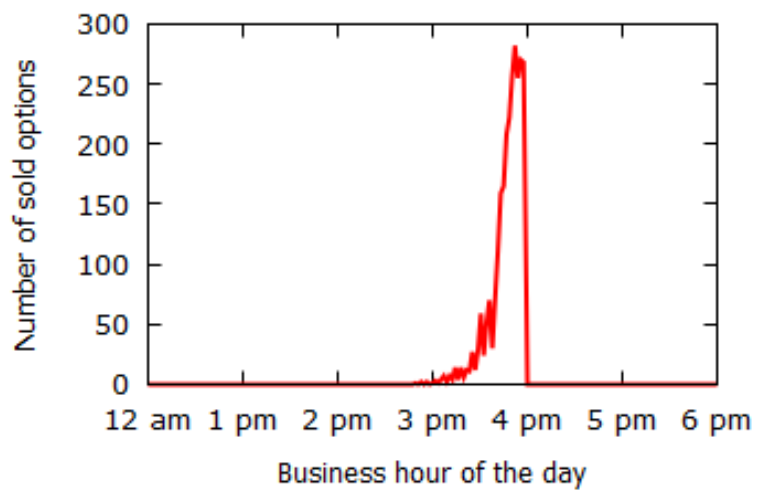

(a) $(0, N)$ case
Frequency of sold options

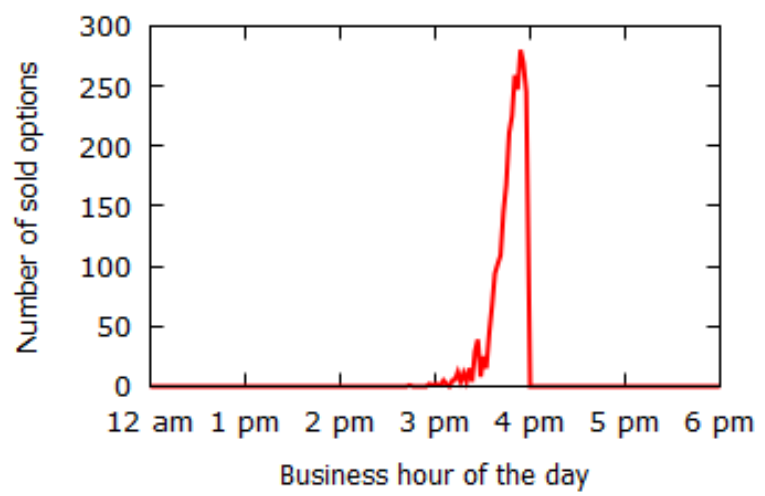

(b) $(1, N)$ case

Figure 4: Simulation with using 5000 random sample paths focusing on the time point of the day when options are sold under Case (i) for parameter combination I where the second time interval is between $4 \mathrm{pm}$ and $6 \mathrm{pm}$, thus $T=6 \mathrm{pm}$ is the end of the business day. On the graph we focused on the business hours when real interactions happen. The contract values for the simulations belong to the one we receive with using 20000 days in computation. The initial imbalance value is 0 for all simulations and we applied grid choice A.

\subsection{Numerical results for Case (ii)}

Now we consider Case (ii) where options can only be sold at the beginning of the business day. This constraint simplifies the possible decisions of the storage operator by vastly reducing the available strategies the storage operator can undertake during the day. At the same time, with this constraint we can successfully rule out the ability of the storage operator to make guaranteed profits by selling options that will not be exercised.

For this Case (ii) we provide a comprehensive analysis, presenting the calculated real values for both parties as well as the optimal strategies for the storage operator. Firstly, in Figure 5 we analyse the real value against a progressively longer terminal time and see that the solutions will converge, and the rate is similar to that in Figure 2. This means that we can again accept the result after 20000 computation days as a good approximation to the lifetime value using our fixed annual interest rate $r=0.1$. An investigation concerning the effect of changing the interest rate can be seen in Appendix D.

Table 3 reveals the percentage of sold and exercised options for different parameter combinations. Among these, the premium prices are varied, whilst all other parameters remain fixed. We can see that under combination III the storage operator would not sell any of the two options at the beginning of the day, however under combination I where the premium is $£ 5$ higher they would sell both the options with only one exception when the imbalance is -300 and the battery is full at the beginning of the day. For combination IV, where the premium is even higher, they would sell both the options for all discussed cases. It is important to note that in Case (ii), we only have two values of sold percentages, namely $0 \%$ and $100 \%$. The storage operator makes a decision at the day beginning about whether it is optimal or not to sell an option to the system operator. The storage operator can choose instead to profit by trading on the electricity market, and in the case when the realised aggregated profit by undertaking option activities is less than this former profit, then the storage operator simply never sells an option. We can infer that this must be the case under combination III. Once the premium is large enough to compensate the profit of the market trading activities then the aggregated profit by undertaking option activities outstrips this former profit and so the storage operator will always sell an option at the beginning of the day. This is what appears to be the case under combinations I and IV. We can also see that the percentage of exercised options does not depend 
significantly on the initial imbalance and this value is close to $60 \%$ in every case. This is because of the high mean reversion of the SDE in this case, since a surplus (or deficit) of demand at $t=0$ has almost no influence on whether there is a surplus (or deficit) for $t \in\left[t_{2}, T\right]$.

In Figure 6 we can see how the real values for both parties change if we vary the length of the first and second periods. As the hours available for exercise $\left(T-t_{2}\right)$ increases the likelihood that options get exercised also increases and so the real value of the system operator increases, whereas the value for the storage operator decreases. We also see that the sum of the individual values is the highest if the length of the second period is 1 hour and the lowest if the length of the second period is 2 hours. We also see that if the length of the period is larger than 2 hours then the value of the system operator is 0 , indicating that no options are traded between the two parties. As the aim of this study to examine option trading activities we can exclude these cases from further analysis.

Figure 7 illustrates the change in the premium price, as we present the real values using 20000 simulated days for both parties under different parameter combinations. In this figure we can see that under low premium price (5 and 10) combinations the storage operator has a small positive real value and the system operator has zero real value, since no options are traded and the only value for the storage operator is in trading on the market. For higher premium prices (15, 20 and 25) the real value of the system operator decreases with increasing premium prices, whereas the real value of the storage operator increases with increasing premium prices, while the sum also slightly decreases, but appears to be the same for premium prices 20 and 25. Under these two latter parameter combinations, options are actually sold under any reasonable imbalance value as we can see in Table 3 and as is further confirmed by Figure 8, whereas for premium price 15 options are sold for a limited initial imbalance range especially call options. This latter figure informs us that options are essentially always sold under these parameter combinations, barring some exceptionally large or small imbalance values. The constant sum for 20 and 25 premium prices together with the $100 \%$ sold options ratio in Table 3 indicate that only the proportion of the positive sum between the two parties is shifted among these different premium price combinations. They can exploit a constant positive profit by having themselves undertaken in option trading activities, in which the profit is actually smaller than the one that is available for the storage operator by solely trading on the electricity market. Even though the sum is smaller, it is still positive, and as these are the cases when options are really traded between the two parties, therefore these are in line with the objective of this work. This constant positive profit can later be redistributed between the two parties and so we can assess that the option selling activities can be beneficial for both parties. As we have stated, according to BSIS in the UK there is a 70\%-30\% share rule regarding the share of the over-, and underspending of the system operator. Our case is a little different, since we have not only calculated the value for the system operator but also for the storage operator who is another member of the industry. Since the optimal decisions are made from the storage operator's point of view, and the system operator always willingly accepts the offered options it is obvious that the storage operator should provide a rebate for the system operator in return for their compliant behaviour. The amount of this rebate can be negotiated between the two parties, but it is an obvious requirement towards the storage operator to share sufficient profit to make the system operator final value at least positive. This means that we are interested in maximising the sum of the individual party's profits, which positive sum indicates a social welfare situation and will be later shared between the two parties at a rate that is acceptable for both of them.

One interesting outcome from our numerical solution is that we are able to present the optimal operational strategy of the storage operator at the beginning and the end of the business day for the possible initial states that are $(0, \mathrm{~N})$ and $(1, \mathrm{~N})$ in Figures 8,9 and 10 . Throughout these calculations we apply grid choice B (see Table 5 in Appendix B) so that the positions at which a decision is made can be found more accurately. In subfigures (a) and (b) of Figures 8, 9 and 10 we indeed present the optimal strategy for start of the business day when the storage operator can possibly sell an option; whereas in subfigures (c) and (d) of Figures 8 . 9 and 10 we present them for the end of the business day that happens before the first time point of the upcoming business day. The strategies presented in (c) and (d) give information as to the state (empty or 


\begin{tabular}{lllll}
\hline & \multicolumn{1}{c}{ Initial imbalance $X(0)$} & $\mathbf{- 3 0 0}$ & $\mathbf{0}$ & $\mathbf{3 0 0}$ \\
\hline \multirow{5}{*}{ Combination III } & Frequency of bought options initial state $(1, N)$ & $0 \%$ & $0 \%$ & $0 \%$ \\
& Frequency of exercised options initial state $(1, N)$ & $0 \%$ & $0 \%$ & $0 \%$ \\
& Frequency of bought options initial state $(0, N)$ & $0 \%$ & $0 \%$ & $0 \%$ \\
& Frequency of exercised options initial state $(0, N)$ & $0 \%$ & $0 \%$ & $0 \%$ \\
\hline \multirow{5}{*}{ Combination I } & Frequency of bought options initial state $(1, N)$ & $0 \%$ & $100 \%$ & $100 \%$ \\
& Frequency of exercised options initial state $(1, N)$ & $0 \%$ & $59 \%$ & $59 \%$ \\
& Frequency of bought options initial state $(0, N)$ & $100 \%$ & $100 \%$ & $100 \%$ \\
& Frequency of exercised options initial state $(0, N)$ & $59 \%$ & $59 \%$ & $59 \%$ \\
\hline \multirow{5}{*}{ Combination IV } & Frequency of bought options initial state $(1, N)$ & $100 \%$ & $100 \%$ & $100 \%$ \\
& Frequency of exercised options initial state $(1, N)$ & $59 \%$ & $59 \%$ & $59 \%$ \\
& Frequency of bought options initial state $(0, N)$ & $100 \%$ & $100 \%$ & $100 \%$ \\
& Frequency of exercised options initial state $(0, N)$ & $59 \%$ & $59 \%$ & $59 \%$ \\
\hline
\end{tabular}

Table 3: A table containing the results of the simulations. Using these simulations we calculated the frequencies at which options are sold and exercised on a given day under Case (ii) for different parameter combinations and initial parameters applying grid choice A. The second time interval is between $4 \mathrm{pm}$ and $6 \mathrm{pm}$, thus $T=6 \mathrm{pm}$ is the end of the business day. Repetition of days is performed up to 20000 and the sample size is 5000 for each calculation. The frequencies are rounded to the nearest integer.

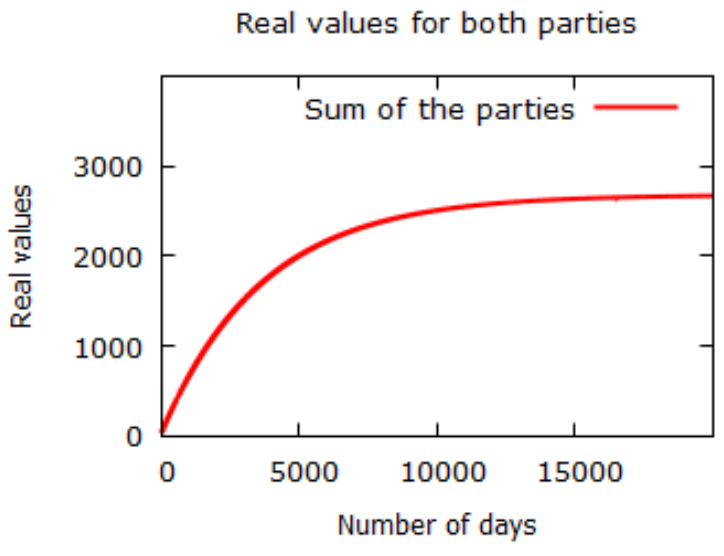

(a) Individual values

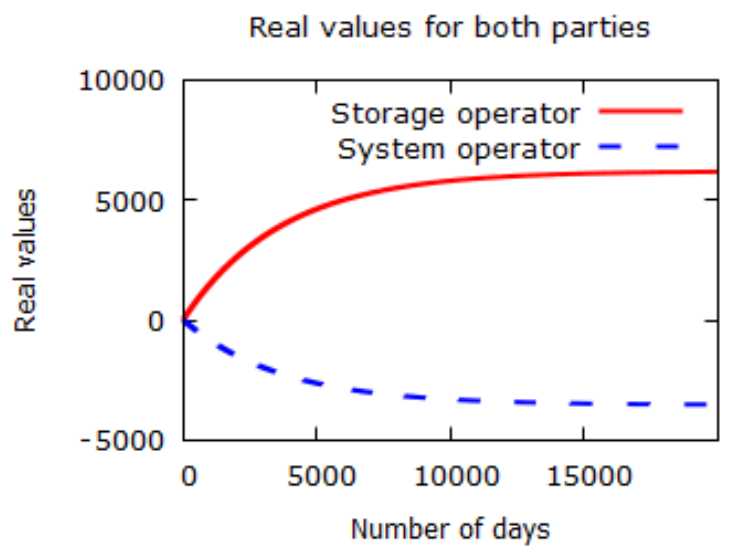

(b) Sum value

Figure 5: Corresponding real values to Case (ii) under parameter combination I using grid choice A against increasing numbers of days. The contract values denote the value in the beginning of the business day under 0 imbalance value and for initial case $(1, N)$. The second time interval is between $4 \mathrm{pm}$ and $6 \mathrm{pm}$, thus $T=6 \mathrm{pm}$ is the end of the business day. 


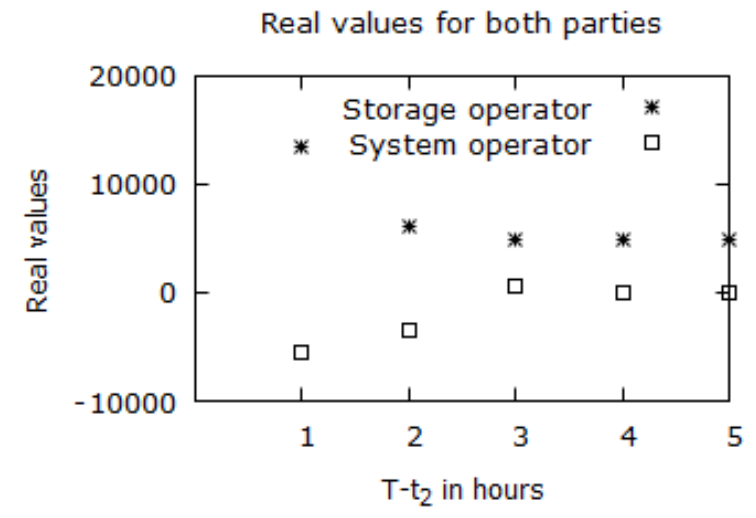

(a) Individual values

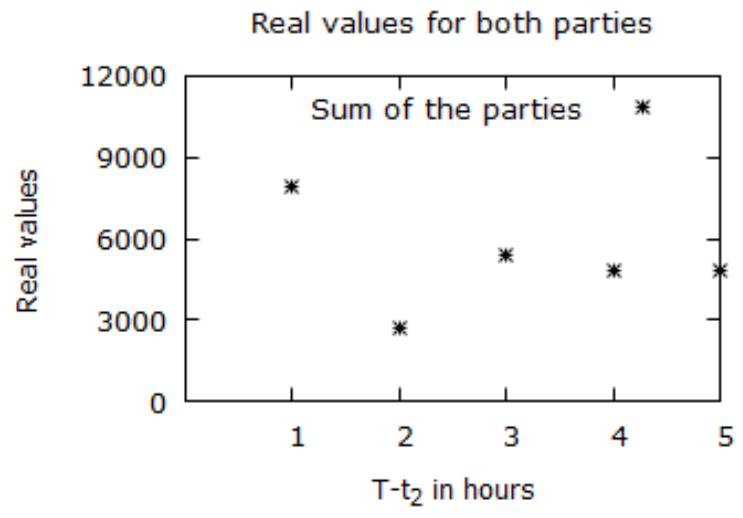

(b) Sum value

Figure 6: The change of corresponding real values on the number of hours to exercise the options to Case (ii) under parameter combination I. The first and second period cover varying amount of hours, however $T=6 \mathrm{pm}$ is fixed as the end of the business day for all examples. The contract values denote the value in the beginning of the business day under 0 imbalance value and for initial case $(1, N)$ after 20000 repeated days using grid choice A.

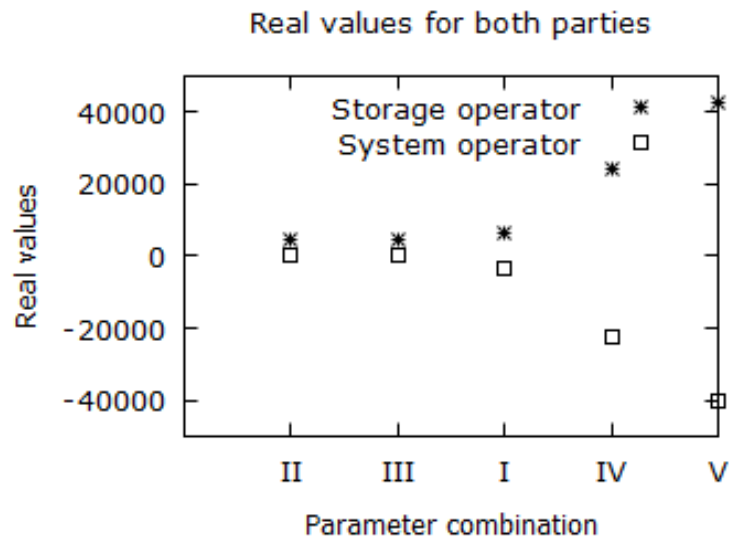

(a) Individual values

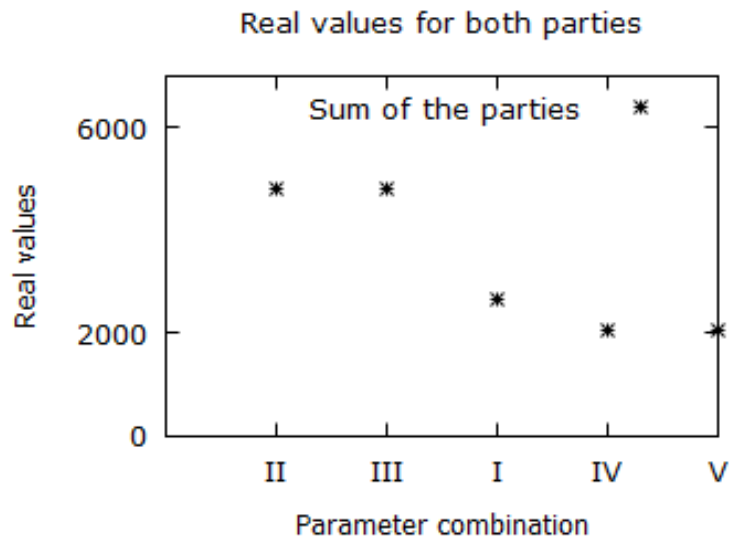

(b) Sum value

Figure 7: The change of corresponding real values on the premium prices to Case (ii) by using different parameter combinations. The second time interval is between $4 \mathrm{pm}$ and $6 \mathrm{pm}$, thus $T=6 \mathrm{pm}$ is the end of the business day. The contract values denote the value in the beginning of the business day under 0 imbalance value and for initial case $(1, N)$ after 20000 repeated days using grid choice A. 
full) the storage operator seeks to achieve so that they are able to sell an option to the system operator (if they wish to). These subfigures also reveal a sensible fact that in general the storage operator tends to sell electricity on the electricity market under smaller imbalance values and tends to buy under higher imbalance values. This is reassuring since the electricity price on the market is a decreasing function of the actual imbalance. We have calculated these optimal decisions for a range of different parameter combinations to verify the strategy as optimal and identify interesting patterns.

We have already discussed some properties of Figure 8 , in which we vary premium prices. In this figure, the parameter combinations are presented in order of increasing premium prices: II, III, I, IV, V. We can see from the strategies that options are not sold at all for lower premium price combinations (II and III). As the premium prices increase above 15, the imbalance region to sell an option also increases. This is reassuring, since the storage operator receives the premium and so they are more likely to sell an option if this premium is higher. Furthermore, it is important to note that the storage operator stays in state $(0, \mathrm{~N})$ or $(1, \mathrm{~N})$ for all imbalance values at the end of the business day for parameter combination II and III as seen in (c) and (d) of Figure 8 . This phenomenon indicates that since there is no option selling at the subsequent time point whatsoever, the storage operator is not interested in changing the state solely for placing the battery in an appropriate state where optimal option selling or buying is possible at the next time point. Furthermore, since the electricity price is time dependent it is reasonable to assume that there exist time points at other times of the day when it is more appropriate to buy or sell electricity on the market simply for profiteering reasons. Thus, there is no real incentive to change the state of the battery at the end of business day for these parameter combinations.

In Figure 9 we can see how a change in strike price affects the optimal strategies. In the case of the put option, the strike price is to be paid by the storage operator whenever an exercise happens, hence it is beneficial for them to have a lower $K_{p}$ value. Conversely, for the case of a call option, the strike price will be paid to the storage operator whenever an exercise happens, so it is beneficial for them to have a higher $K_{c}$ value. In this figure, we order the parameter combinations by increasing (decreasing) values of $K_{p}$ $\left(K_{c}\right)$ : VI, VII, I, VIII, IX. Therefore, we expect that this is the order of preference for the storage operator towards selling the actual option. This is indeed confirmed in Figure 9 since the highest range to optimally sell options is found for combination VI and the smallest range is found in combination IX.

In Figure 10 we can see how a change in the fixed exercise strategies $x_{p}^{*}, x_{c}^{*}$ affects the optimal strategy of the storage operator. In this figure, the parameter combinations are in order of increasing absolute magnitude of the fixed imbalance price: X, I, XI, XII, and XIII are $x_{p}^{*}=100,200,300,400$ and 500 respectively. For the storage operator it may not be beneficial to have a large number of exercised options, since each time the option is exercised the storage operator may face a financial loss. We will find that a lower exercise rate is expected for higher values of $x_{p}^{*}$, since the imbalance value trivially less often crosses these values, triggering exercise. This claim is confirmed in Figure 10 since the parameter combinations with a higher range to optimally sell options (making selling more likely) correspond to higher fixed imbalance values.

We have discussed some exceptional parameter combinations such that the storage operator always stays in the given state at the end of the business day. Apart from these cases, we can see in subfigures (c) and (d) of Figures 8, 9 and 10 that the change on the aforementioned option-related parameters does not really make an impact on the values separating states $(0, N)$ and $(1, N)$. This indicates that the storage operator might make a change to the state of the battery to make it available for optimal sale or optimal buy, however they also take into account the cost of this change, that is determined by the electricity price. This electricity price, however, only depends on the current imbalance value and the actual time of the day and does not depend on any option-related parameter, implying that placing the battery into a different state incurs the same cost for the storage operator for all of the chosen parameter combinations. Finally, as we can see in subfigures (c) and (d) of Figures 8, 9 and 10 the storage operator sells electricity on the liquid market for lower negative imbalance values and buys electricity on the liquid market for higher positive imbalance values. The reason behind this is that the electricity price is a decreasing function of the imbalance value and so a higher imbalance value implies a cheaper price and vice versa. Furthermore, these strategies about acting on the liquid market also imply balancing actions on their own. The fact that the storage operator is only willing to empty the battery for negative imbalance values and is only willing to charge up the battery 
for positive values is beneficial for the system as by undertaking these actions imbalances are also corrected and the system is transferred into a more stable state.

To sum up Case (ii), we have provided parameter combinations IV and V where options are intensively traded between the two parties. Further, a mutual benefit is indeed available for these combinations indicating that the optimal decision of the storage operator will result in a positive profit for them and at the same time the system operator can achieve a lower balancing cost compared to the target short-term one. This mutual benefit can be maximised by choosing the parameter combination that results in the largest sum of individual real values. In our analysis, however, the total profits are approximately equal for parameter combinations IV and V. As we can see in Table 1 all these combinations satisfy the sustainability conditions, meaning that both parties are willing to enter into the agreement under the given fixed parameters. On the other hand, as we have stated earlier the incentive for the system operator to be involved in this agreement is to receive a long-term balancing tool by reducing some of the financial and operational risks in power-system balancing. Such a tool is clearly provided with these combinations. By using high enough premium prices we can realise a $100 \%$ sold options ratio by Case (ii) which is larger than any sold options ratio by Case (i), thus we can be in accordance with the utmost motivation of this paper to provide balancing tools for the system operator.

\section{Conclusions}

As renewable energy sources become more prevalent, the transmission system operator has to pay increasing attention to satisfy the need for balancing the demand and supply sides of the electricity market. There are increasing concerns related to short-term balancing as a consequence of the transition from the more flexible traditional sources of energy to inflexible intermittent renewable energy sources. To guard against these concerns, we propose in our work that a third-term party, namely the storage operator, can offer flexibility for the market by trading in financial options.

We have carefully prescribed the details of our proposed agreement. This contract involves options with electricity as the underlying physical asset, where the price of electricity can be determined by a daily periodic function and the current imbalance level. We restrict the possible exercise time of the offered options to the end of the business day which in our case is fixed to be $6 \mathrm{pm}$. We have compared two different cases that differ in time periods at which options are available to be sold and have shown that one of these has superior properties. We have then shown that the contract belonging to this more advantageous Case (ii) can indeed be applied as a balancing cost reduction alternative for the system operator. Further, even allowing for the selfish optimal decisions of the battery owner, a financial profit is available for both parties. This mutual benefit possibility makes this contract attractive for both parties. The financial profit for the storage operator simply makes the storage facility economically favourable for its owner to have taken part in this contract. We can see that with certain parameter combinations, the balancing costs that emerge from this contract are lower than the so called target balancing costs (see [3]) which is about acting on the short-term (managed) spot market; this is a cost reduction for the system operator. A further advantage of this contract is to act as insurance by transferring some short-term balancing duties to the prescribed agreement and so guard against unforeseeable economic circumstances.

The work was performed with using empirical data from the UK market. With the help of this data, we stochastically modelled the imbalance value and inferred the electricity price from this process. After establishing an appropriate optimal control scheme using [12, we managed to express our optimal switching problem with the help of a free boundary problem. One of the main contributions has been to numerically calculate the lifetime contract values for both parties having solved the corresponding free-boundary problem. By using a fixed positive constant interest rate $r=0.1$ we have shown that this lifetime contract value is indeed bounded for both parties. It is important to note that the analysis carried out in this paper is not necessarily UK specific and the calculations could be replicated using imbalance and price data from different countries, if it is available. However, different challenges will present themselves when the analysis is applied to different markets. The UK energy market is one of the most advanced deregulated markets, therefore we have more flexibility to propose contracts like the one we did in this work. Furthermore, the geography of 
Optimal Strategy at the day beginning

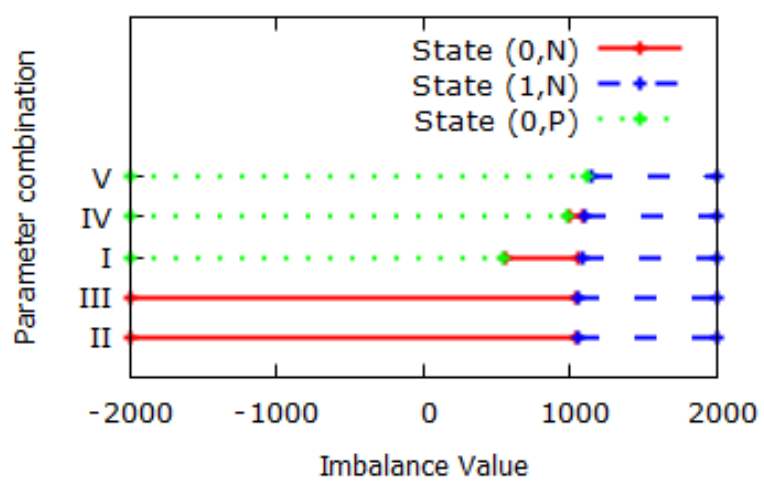

(a) $(0, \mathrm{~N})$ case

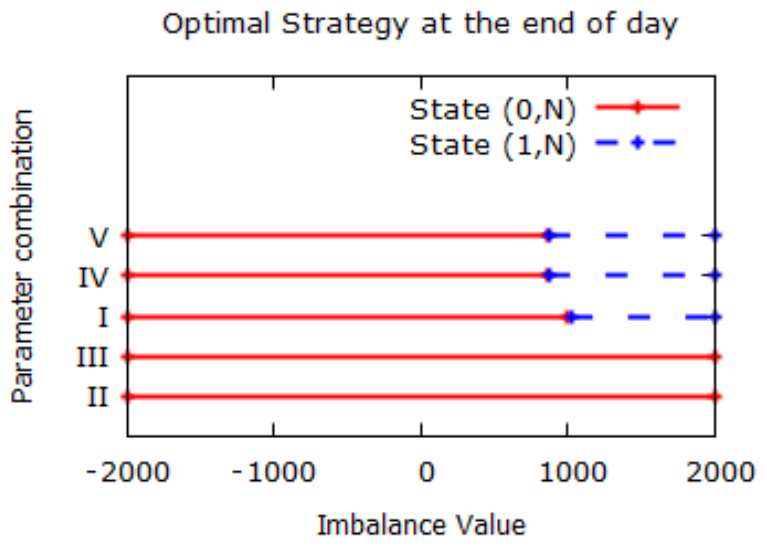

(c) $(0, \mathrm{~N})$ case
Optimal Strategy at the day beginning

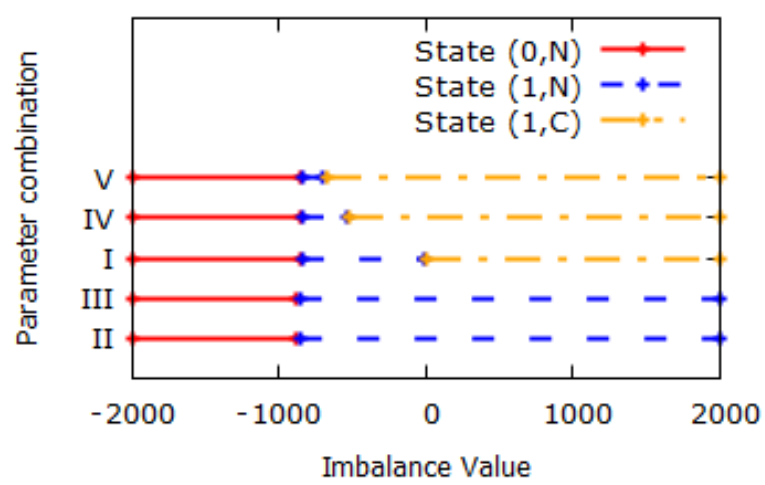

(b) $(1, \mathrm{~N})$ case

Optimal Strategy at the end of day

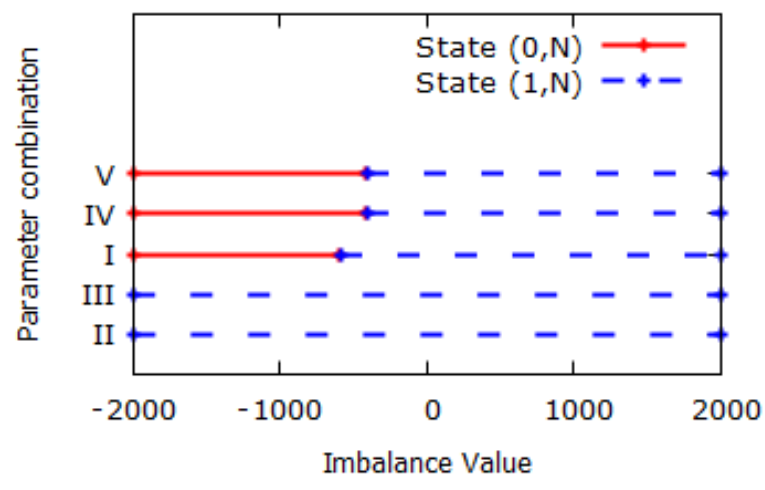

(d) $(1, \mathrm{~N})$ case

Figure 8: Optimal operational strategy in the business day beginning in subfigures (a) and (b) and in the end of the business day in subfigures (c) and (d) for different parameter combinations that vary the premium prices over the imbalance horizon under Case (ii) where the second time interval is between $4 \mathrm{pm}$ and $6 \mathrm{pm}$, thus $T=6 \mathrm{pm}$ is the end of the business day. Computed number of days for this contract is 500 and we applied grid choice B through the calculations. 
Optimal Strategy at the day beginning

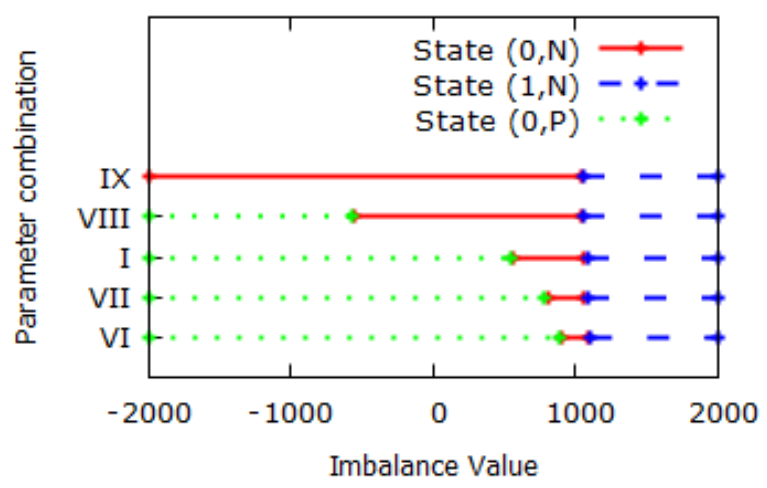

(a) $(0, \mathrm{~N})$ case

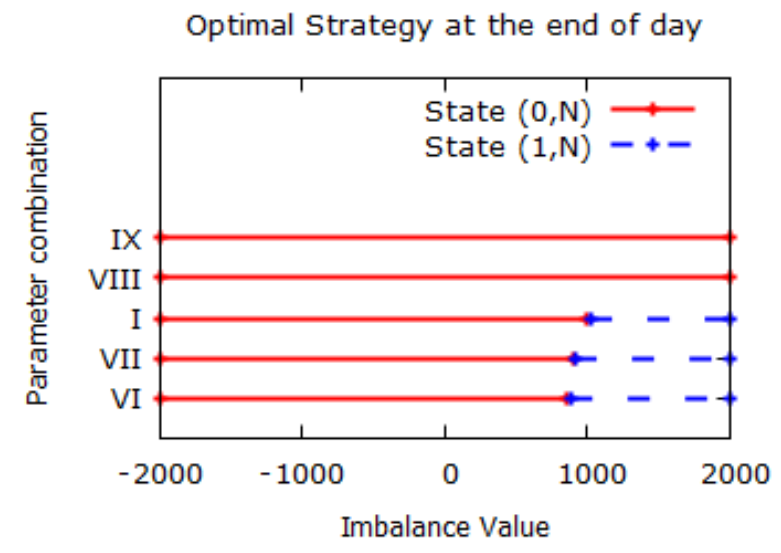

(c) $(0, N)$ case
Optimal Strategy at the day beginning

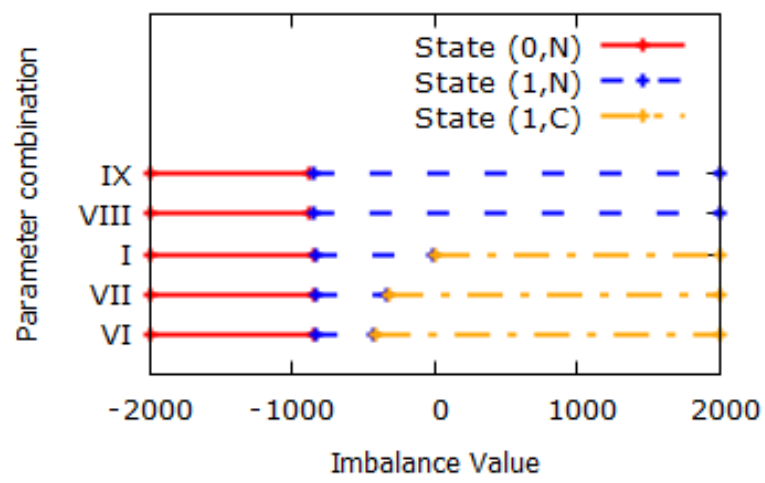

(b) $(1, N)$ case

Optimal Strategy at the end of day

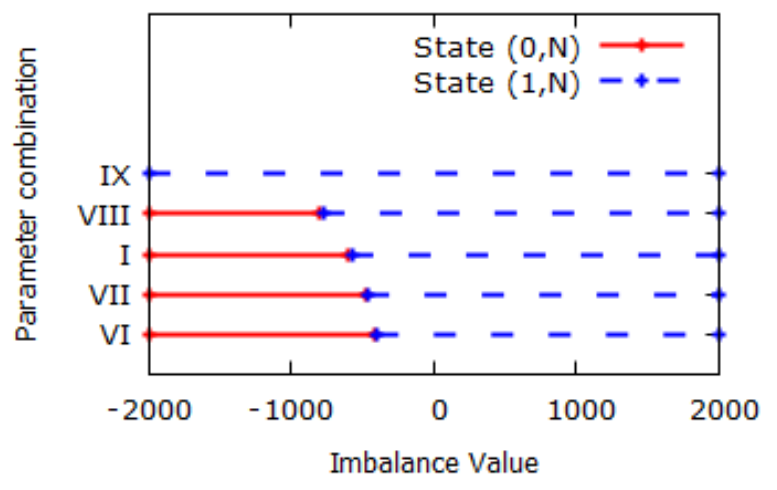

(d) $(1, \mathrm{~N})$ case

Figure 9: Optimal operational strategy in the business day beginning in subfigures (a) and (b) and in the end of the business day in subfigures (c) and (d) for different parameter combinations that vary the strike over the imbalance horizon under Case (ii) where the second time interval is between $4 \mathrm{pm}$ and $6 \mathrm{pm}$, thus $T=6 \mathrm{pm}$ is the end of the business day. Computed number of days for this contract is 500 and we applied grid choice B through the calculations. 
Optimal Strategy at the day beginning

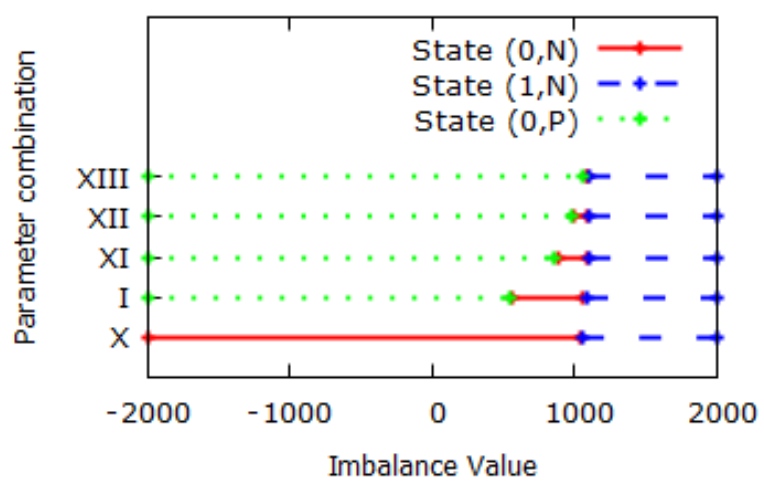

(a) $(0, \mathrm{~N})$ case

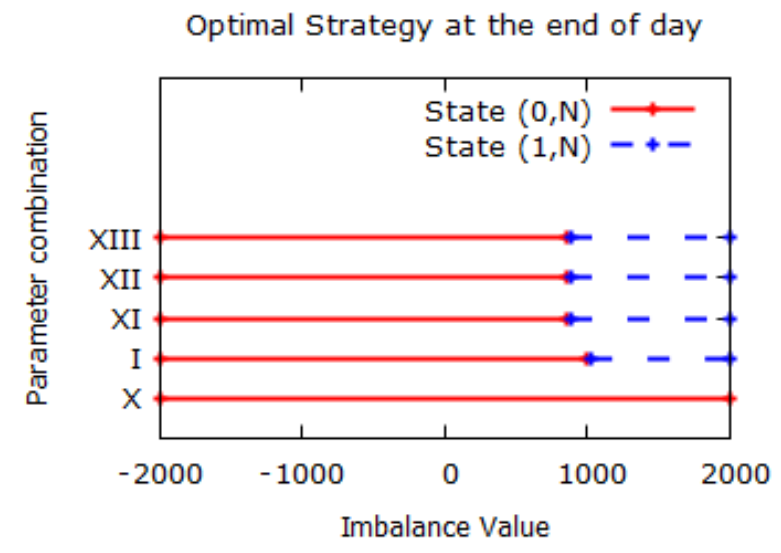

(c) $(0, N)$ case
Optimal Strategy at the day beginning

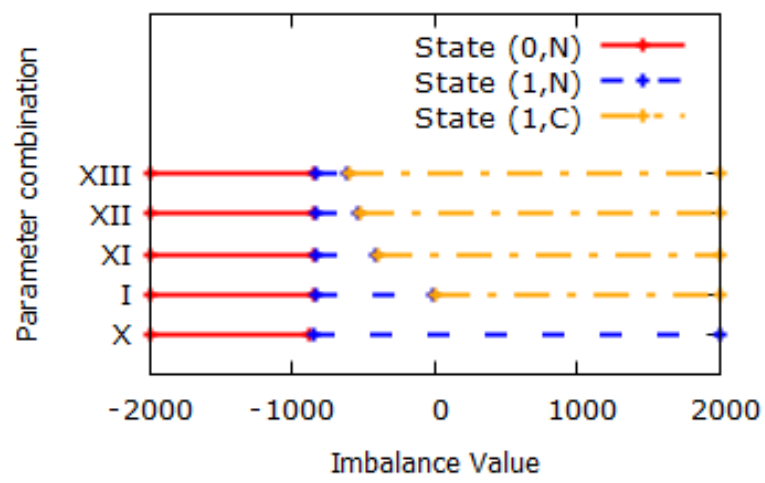

(b) $(1, N)$ case

Optimal Strategy at the end of day

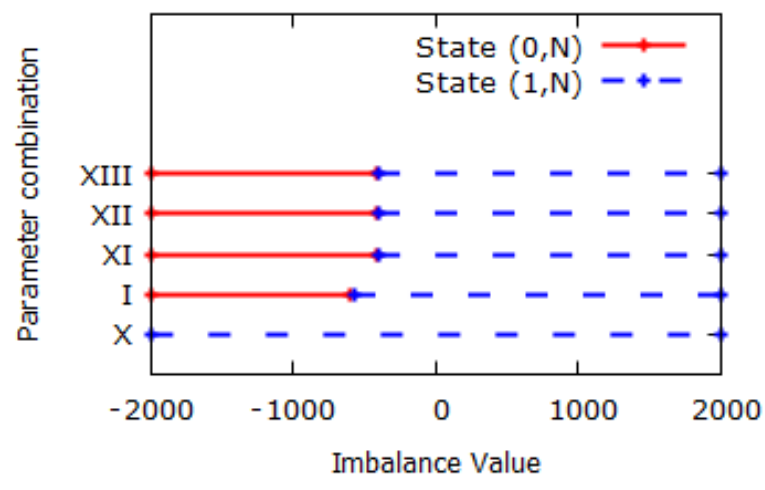

(d) $(1, \mathrm{~N})$ case

Figure 10: Optimal operational strategy in the business day beginning in subfigures (a) and (b) and in the end of the business day in subfigures (c) and (d) for different parameter combinations that vary the fixed thresholds over the imbalance horizon under Case (ii) where the second time interval is between $4 \mathrm{pm}$ and $6 \mathrm{pm}$, thus $T=6 \mathrm{pm}$ is the end of the business day. Computed number of days for this contract is 500 and we applied grid choice B through the calculations. 
the UK's location (as an island) means that it is less connected to other grids and is more likely to suffer short term imbalances than other more well connected grids. It would be interesting to see how this analysis would fit into different countries where adequate balancing corrections are still becoming very important.

A fixed threshold strategy of the system operator and their unconditional willingness to enter into the contract were used as simplifying assumptions during the work. We could have allowed the system operator to have more flexibility in whether or not they buy the option. One way to include this would be to stochastically model the system operator's decision about the acceptance of offered options by incorporating their inclination to accept more favourable options with a higher probability. This would complicate the problem somewhat in terms of adding multiple options and the extra complexity that entails. Another assumption to relax would be the decision strategy employed by the system operator when the option has been bought. By modelling the exercise decision by a jump process, this would lead to a more sophisticated exercise decision model that could make the decision process imbalance dependent.

We hope that this approach can be used to experiment with different contracts that could encourage more storage operators into the market. Storage operator face large capital costs to start operation and as government incentives are reduced more innovative market solutions will be required to ensure the stability and reliability of electricity grids in the years to come.

\section{Acknowledgement}

We express our gratitude to Prof. John Moriarty for many incisive ideas and comments. We would like to thank the anonymous referees for providing extensive and constructive feedback that helped improve this work. 


\section{Appendices}

\section{A. Time dependency of the price function}

Here we analyse the modelling of the electricity price function. We again establish a relationship between the actual physical imbalance value and electricity price such as in [24, 31]. That is, we model the electricity price as a function of the current imbalance volume. We show empirical system prices against the physical imbalance values in the stated time range in Figure 11. We follow [31] in deciding to model the relation between the two variables as a linear function in the first round. We can see the data points and the fitted linear function in Figure $11 \mathrm{a}$. The R-squared value of this linear fit is relatively small $(\approx 0.2)$, however. This means that we can dismiss the linear relation between these variables and seek for an alternative. Therefore, building on the idea that there are 48 settlement periods each day we decided to fit a linear regression separately using data points belonging to just one of these settlement periods. That is, we performed 48 fits and found 48 corresponding interception and slope parameters. We can see the data points belonging to the $46^{\text {th }}$ settlement period in the day and the fitted linear function in Figure $11 \mathrm{~b}$. The R-squared values of the 48 different fits increased significantly $(\approx 0.4)$ implying that the this new model is superior to the linear fit. Figure 12 shows 48 intercept and slope points of the corresponding linear regressions. These plots reveal a clear pattern, namely a periodicity phenomenon that reinforces the necessity of this additional time component.

Furthermore, we see that an appropriate price stack function is of the following form:

$$
f(t, x)=c(t)+b(t) x
$$

where $x$ is the current imbalance volume at $t \quad(0 \leq t \leq 1)$ is the time of the business day. We apply an assumption such that this $f(t, x): \mathbb{R}^{[0,1]} \times \mathbb{R} \rightarrow \mathbb{R}$ function is smooth in both variables, implying that its first and second derivatives both exist.

In order to effectively apply this model to our empirical data points, we can fit a Fourier series for both the 48 intercept and 48 slope points, which can be expressed after a truncation by using only $k$ terms as follows:

$$
c(t)=c+\sum_{i=1}^{k}(I 1(i) \sin (2 \pi i t)+I 2(i) \cos (2 \pi i t)), \quad b(t)=b+\sum_{i=1}^{k}(S 1(i) \sin (2 \pi i t)+S 2(i) \cos (2 \pi i t)),
$$

and $k$ is chosen in order to achieve a strong fit and the $I 1(i), I 2(i), S 1(i), S 2(i)$ parameters are the estimated coefficients from the empirical data. We show the fits in Figure 12 by using the first four terms for both fits $(k=4)$, which appears to be adequate.

Remark A.1. We do not involve a seasonality analysis for the data points. This choice obviously simplifies our model. On the other hand our model is built on a daily periodicity level with providing balancing reserve services on the same day for the system operator when the option is sold. Therefore each day during the year is considered to be equal in our setting with having the same characteristics for the variables.

\section{B. Base parameter combinations}

As we have indicated we can see our 13 different combinations in Table 1 . We choose the parameters to be in line with the electricity price on the UK market with also paying attention whether or not the sustainability conditions we defined in (18) and $(19)$ hold. Using Table 4, which contains some statistical properties about the price function in our exercise region, we also determined that only 3 of these 13 combinations violate at least one of the two sustainability conditions. That is, parameter combination VII and X violate (18), whereas parameter combination VI violates both $(18)$ and $(19)$. Among these combinations we vary one of the parameters with leaving the others fixed. Throughout the work we apply these 13 combinations to perform our comprehensive analysis of the problem. 


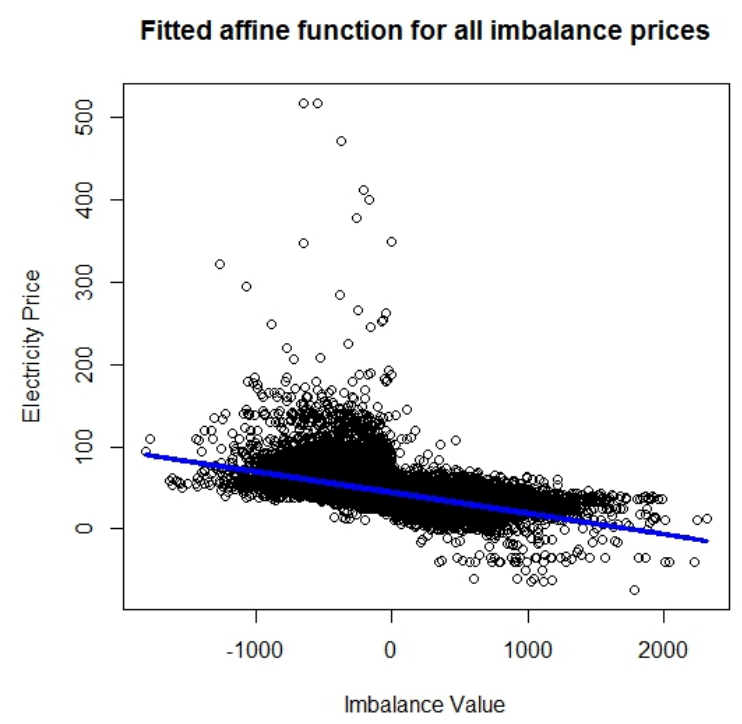

(a)

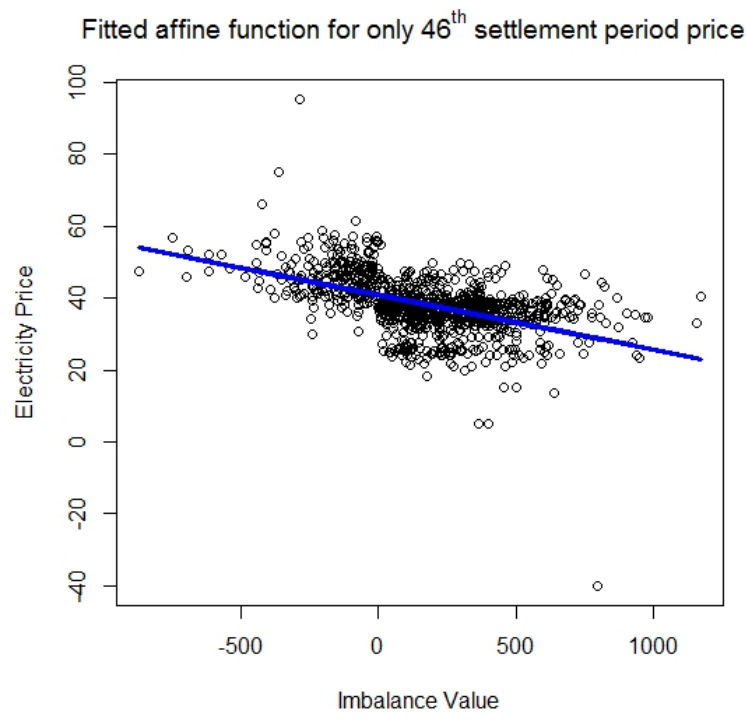

(b)

Figure 11: Empirical data and fitted linear regression in subfigure (a) for the whole data set, and in subfigure (b) for only one settlement period

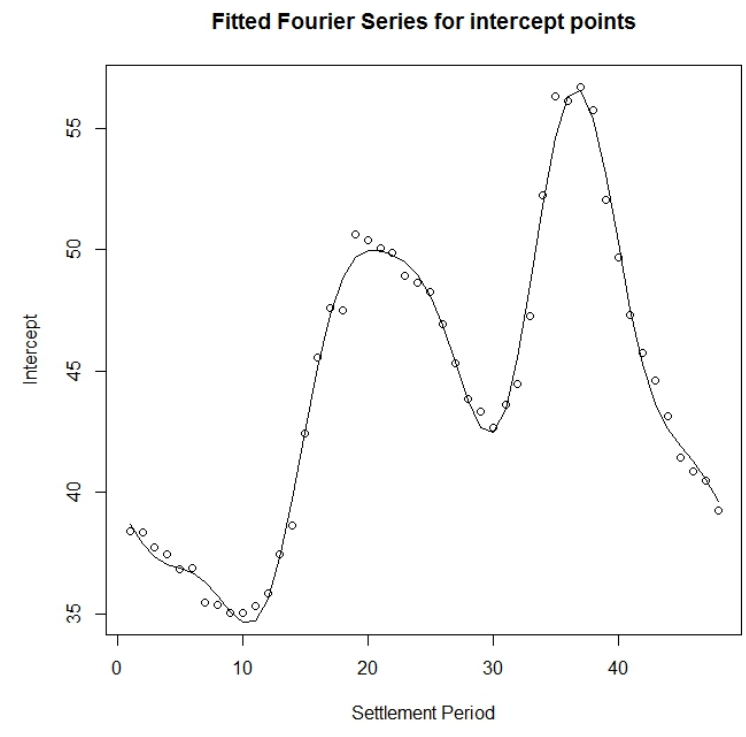

(a)

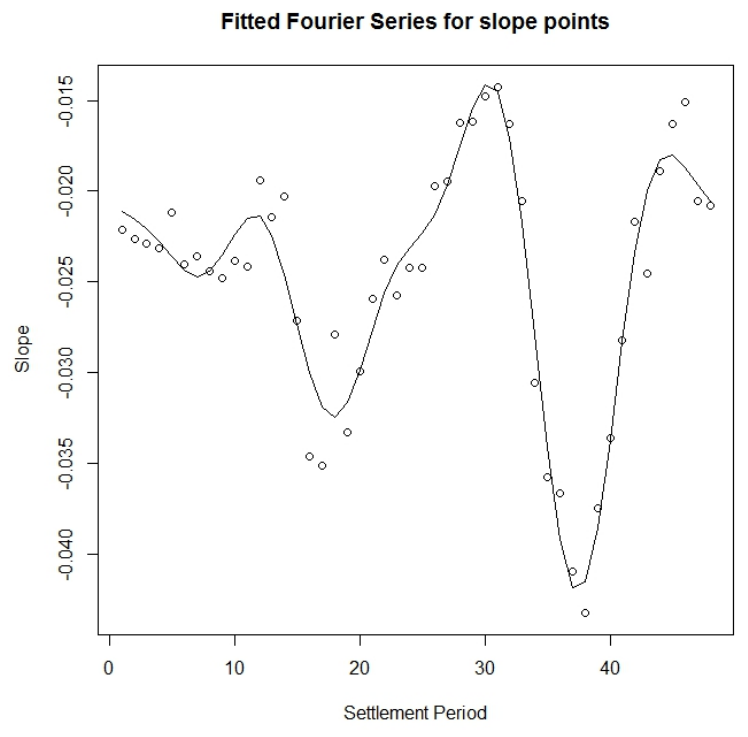

(b)

Figure 12: Fitted Fourier series for the intercept points in subfigure (a) and for the slope points in subfigure (b) of the linear regressions. 


\begin{tabular}{|c|c|c|c|c|c|c|c|c|c|c|c|}
\hline Imbalance $(\mathrm{x})$ & -500 & -400 & -300 & -200 & -100 & 0 & 100 & 200 & 300 & 400 & 500 \\
\hline Largest $f$ in $\left[t_{2}, T\right]$ & 75.71 & 71.82 & 67.92 & 64.03 & 60.13 & 56.24 & 52.34 & 48.45 & 44.57 & 40.94 & 36.76 \\
\hline Smallest $f$ in $\left[t_{2}, T\right]$ & 53.74 & 52.06 & 50.38 & 48.70 & 47.03 & 45.35 & 43.67 & 41.99 & 40.32 & 38.64 & 37.87 \\
\hline Average $f$ in $\left[t_{2}, T\right]$ & 65.11 & 62.35 & 59.59 & 56.83 & 54.07 & 51.31 & 48.55 & 45.79 & 43.03 & 40.27 & 37.51 \\
\hline
\end{tabular}

Table 4: This table shows different values the time- and imbalance dependent price function $f(x, t)$ establishes in the exercise region $\left[t_{2}, T\right]$, where $t_{2}$ is $4 \mathrm{pm}$ and $T$ is $6 \mathrm{pm}$.

\begin{tabular}{|c|c|c|}
\hline Number & jMax & iMax \\
\hline A. & 80 & 800 \\
\hline B. & 200 & 5000 \\
\hline
\end{tabular}

Table 5: Grid combinations.

\section{Numerical scheme}

Now, we describe some details of the means that we numerically solve the optimal switching problem. In our problem we are interested in a two dimensional underlying process $(X, t)$ where $X$ is an Ornstein-Uhlenbeck process and $t$ is simply the time variable. The infinitesimal operator of this joint $(X, t)$ process is of the following form:

$$
\mathbb{L}_{X} V=\frac{\partial}{\partial t} V+\frac{\sigma^{2}}{2} \frac{\partial^{2}}{\partial x^{2}} V-\kappa x \frac{\partial}{\partial x} V
$$

The variational equality for the continuation region reads as:

$$
r \cdot V=\frac{\partial}{\partial t} V+\frac{\sigma^{2}}{2} \frac{\partial^{2}}{\partial x^{2}} V-\kappa x \frac{\partial}{\partial x} V
$$

We can solve the corresponding variational equalities for the continuation region using finite difference methods and then compare the continuation values with the possible stopping values. We follow a conventional manner to perform this (see [29] for example). Clarification is needed, however, on how to model the equations for the smallest and largest imbalances in the continuation region. We disregard the second derivative terms for large negative and positive imbalance volumes since they converge at the quickest rate and so we only analyse the following equations in our numerical calculations by the smallest (jmin) and largest (jmax) imbalance volumes (where $j$ is the imbalance-and $i$ is the time variable):

$$
r \cdot V(j, i)=\frac{\partial}{\partial t} V(j, i)-\kappa x \frac{\partial}{\partial x} V(j, i) \quad \text { for } \quad j=j \min , j \max
$$

We also have to investigate how the number of grid points by the calculations affect the final results. We choose the number of grid points to receive a robust result, but not to make our computation unacceptably lengthy. In Figures 13 and 14 we can see the ratio between the value of two different grid points choice (A and B) for both parties under different premium prices. The values are plotted against the number of days we perform our analysis. We see that these ratios do not deviate far from 1 and so we accept that the choice with smaller number of grid points (A) is a proper choice to perform our numerical calculations throughout the work. The aim of this "grid choice" test is simply to demonstrate that our numerical calculations are robust and there is no need to increase the number of grid points in the finite-difference setting which would mean a longer computational time. That is, we compute our contract values with $j$ Max $=80$ and $i$ Max $=800$ grid points, where $j M a x$ is the number of imbalance grids and $i M a x$ is the number of time grids per day. 
Parameter I: Grid proportion

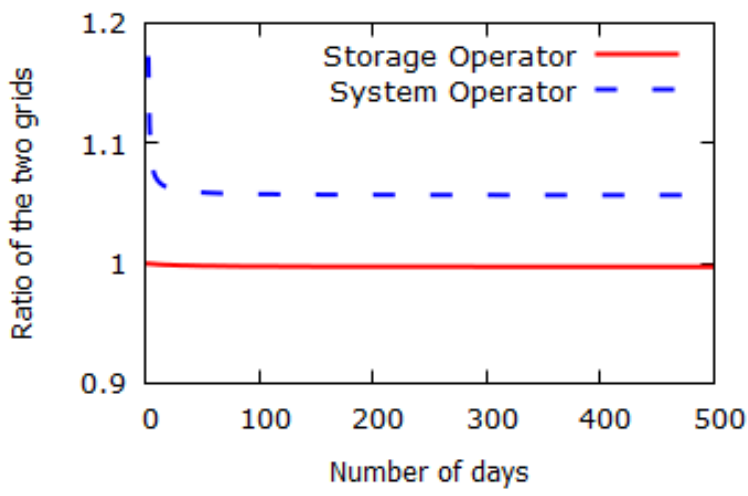

(a) Individual values

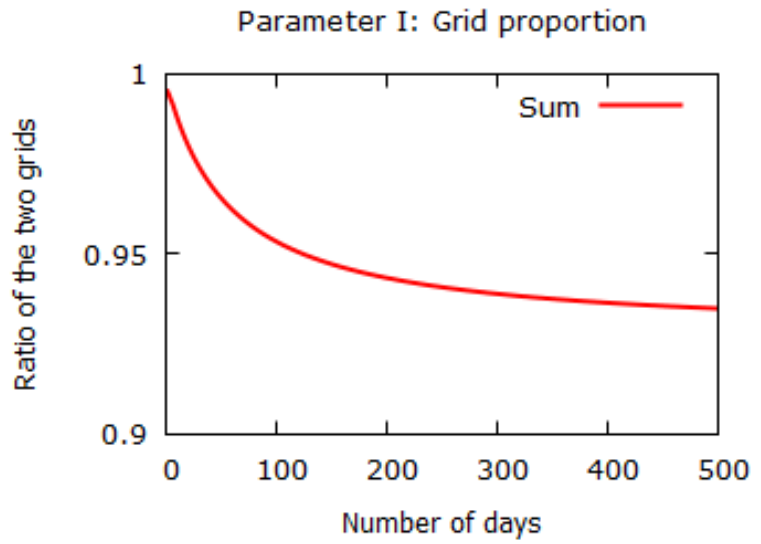

(b) Sum value

Figure 13: The ratio between two real values that are received by dividing the values of grid choice $\mathrm{A}$ with the values of grid choice B element-wise under Case (ii) where the second time interval is between $4 \mathrm{pm}$ and $6 \mathrm{pm}$, thus $T=6 \mathrm{pm}$ is the end of the business day. The contract values denote the value in the beginning of the business day under 0 imbalance value and for initial case $(1, N)$. We compared the results up to 500 days for parameter combination I.

Parameter IV: Grid proportion

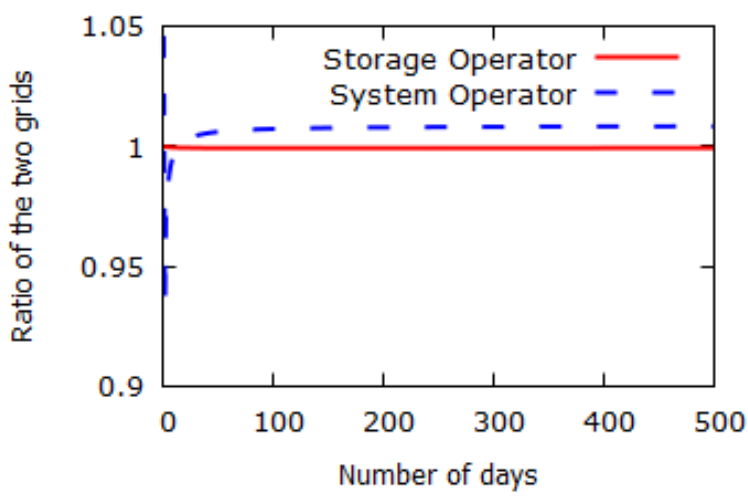

(a) Individual values
Parameter IV: Grid proportion

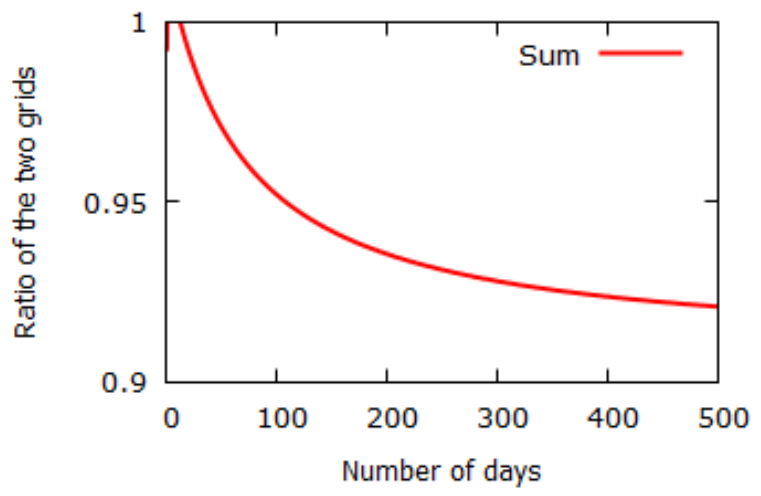

(b) Sum value

Figure 14: The ratio between two real values that are received by dividing the values of grid choice A with the values of grid choice B element-wise under Case (ii) where the second time interval is between $4 \mathrm{pm}$ and $6 \mathrm{pm}$, thus $T=6 \mathrm{pm}$ is the end of the business day. The contract values denote the value in the beginning of the business day under 0 imbalance value and for initial case $(1, N)$. We compared the results up to 500 days for parameter combination IV.

On the other hand, the simulations related to the optimal operational strategy of the storage operator are performed with the more densely covered grid choice $(j M a x=200$ and $i M a x=5000)$ in order to get an even more accurate result. We also have to note that in accordance with the UK empirical data points the smallest and largest imbalance volumes we consider during the calculations are -2000 and 2000, respectively.

\section{Sensitivity of results with respect to changing parameters}

Our aim is now to investigate to what extent the contract results change if we keep varying some of our main parameters. This means that we discuss how a change in the interest rate or in the $C_{2}$ maintenance cost affects our main results and whether or not using the actual $\mu=-94.7489$ mean reversion parameter 
instead of $\mu=0$ strongly affects the final results. Figure 15 reveals how the $C_{2}$ transaction cost affects the contract values for both parties when fixing the rest of the parameters in line with combination I. As discussed, this $C_{2}$ cost is a burden for the storage operator by option exercising times thus our expectation is that if we keep increasing this parameter then the value of the storage operator decreases. This is indeed confirmed in Figure 15. We can also see that the contract value of the system operator is almost constant for low $C_{2}$ values, whereas for $C_{2}=8$ value this value is 0 . This indicates that if the $C_{2}$ cost is large enough then it is not optimal anymore for the storage operator to sell options, therefore no activities happen between the two parties and so we are clearly out of the objective of this paper. Figures 16 17 are meant to investigate how a change in the interest rate affects the contract values and the rate of convergence. These two figures along with Figure 5 indicate that if we decrease $r$ then the rate of convergence is slower, furthermore the corresponding real values are larger. This is due to the fact that the possible future cash-flows are discounted by a smaller constant value and so their actual present values are greater. A comparison between Figure 17 and Figure 5 shows that halving the interest rate approximately doubles both the real values and the number of days to achieve the transient states where values don't increase anymore.

Our last sensitivity analysis is concerned about choosing the most appropriate $\mu$ parameter and confirming that our $\mu=0$ choice in the main part was accurate. In other words, we aim to confirm that replacing the estimated $\mu=-94.7489$ value with $\mu=0$ does not have a significant effect on our most important results. Thus we carry out an analysis concerning the effect of this change for four different parameter combinations in Figure 18. This figure shows that the largest proportion between the values of the two settings can be experienced under combination I, whereas for combinations VI, XIII and V the proportions are a lot smaller. Under the three latter combinations the ratio of the corresponding individual values under the two different $(\mu=-94.7489, \mu=0)$ settings are very close to 1 . We also know from Figures 810 that under these three combinations the option exercise region is very broad meaning that for all reasonable imbalance values options are exercised. On the other hand under combination I this region is relatively narrow, especially under initial case $(1, N)$. Thus we can assess that for cases which are in line with our main objective-to have our two parties be engaged in option trading activities-the actual effect of replacing the actual estimated $\mu$ value with 0 and so by assuming that we have 0 -reverting processes is not very significant. This means that if the storage operator uses the storage facility to primarily offer options for the system operator then our numerical setting with the $\mu=0$ assumption is quite stable. Allied with the fact that we want to build a framework when more options are traded and so the imbalance is more likely to be 0 we can claim that $\mu=0$ assumption does not severely violate our most important results. 


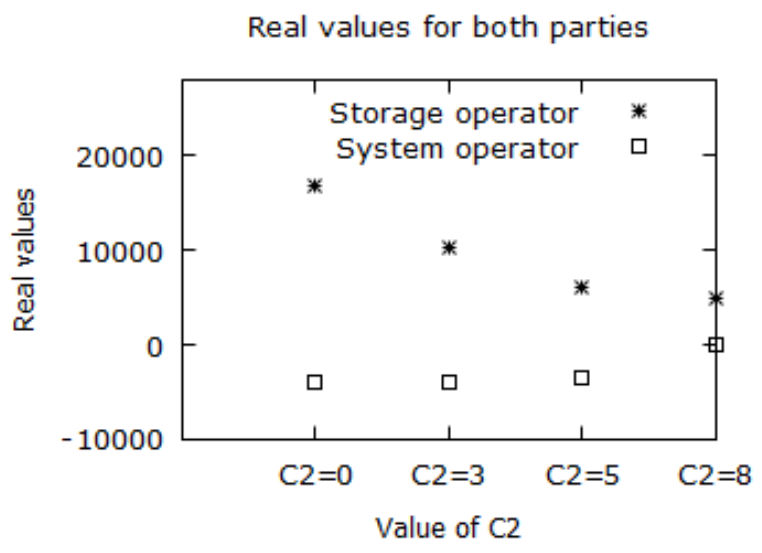

(a) Individual values

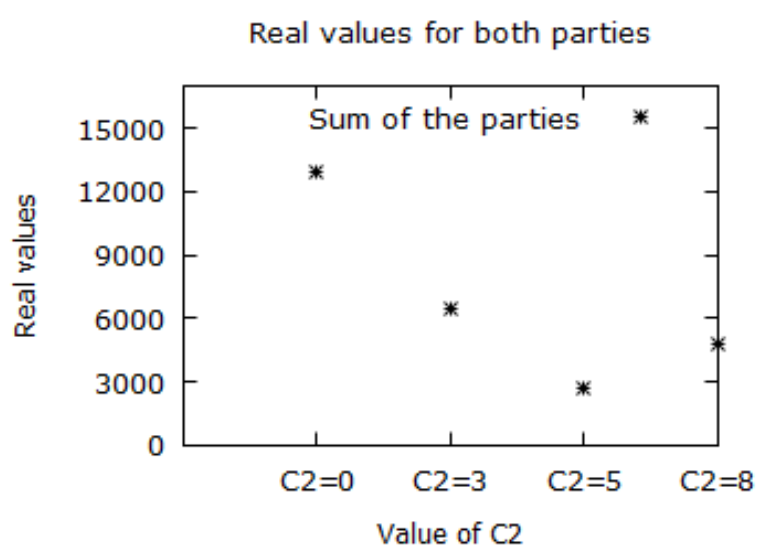

(b) Sum value

Figure 15: Real values using different $C_{2}$ values under Case (ii) where the second time interval is between $4 \mathrm{pm}$ and $6 \mathrm{pm}$, thus $T=6 \mathrm{pm}$ is the end of the business day. The parameter combination we use here is essentially I, meaning that we keep changing $C_{2}$, but the rest of the parameters coincide with that of combination I in Table 1 The contract values denote the value in the beginning of the business day under 0 imbalance value and for initial case $(1, N)$. We compared the results using grid choice $\mathrm{A}$ and applying 20000 days.

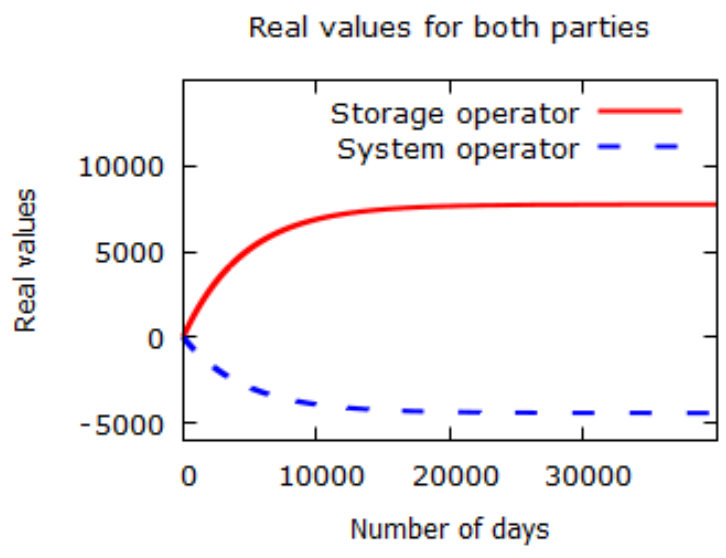

(a) Individual values

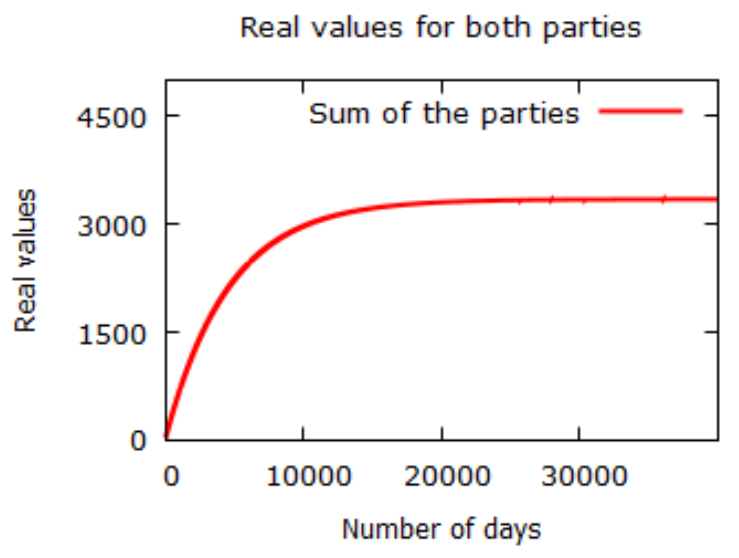

(b) Sum value

Figure 16: Corresponding real values to Case (ii) under essentially parameter combination I, meaning that $r=0.008$ but the rest of the parameters coincide with that of combination I in Table 1 using grid choice A against increasing numbers of days. The contract values denote the value in the beginning of the business day under 0 imbalance value and for initial case $(1, N)$. The second time interval is between $4 \mathrm{pm}$ and $6 \mathrm{pm}$, thus $T=6 \mathrm{pm}$ is the end of the business day. 


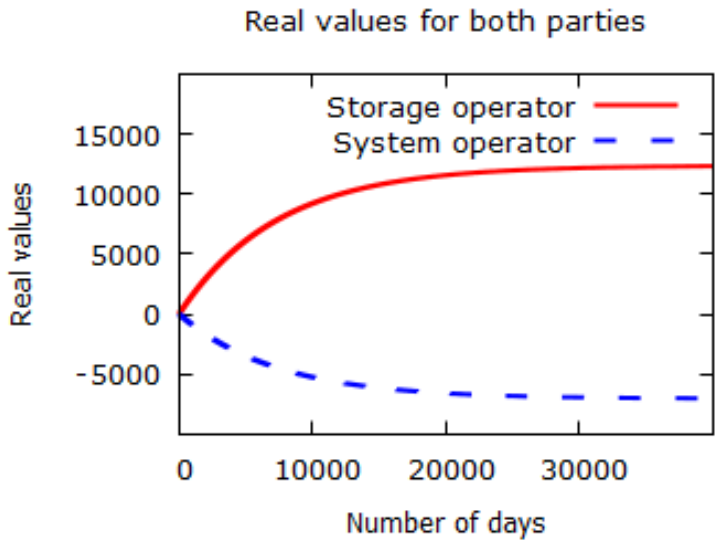

(a) Individual values

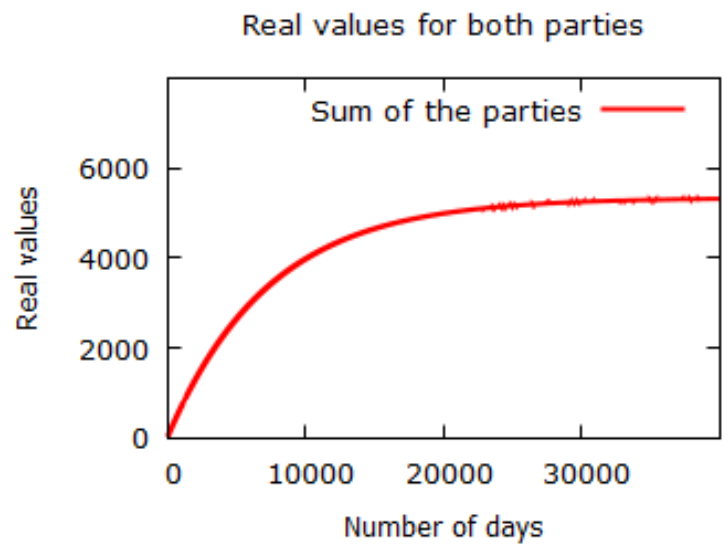

(b) Sum value

Figure 17: Corresponding real values to Case (ii) under essentially parameter combination I, meaning that $r=0.005$ but the rest of the parameters coincide with that of combination I in Table 1 using grid choice A against increasing numbers of days. The contract values denote the value in the beginning of the business day under 0 imbalance value and for initial case $(1, N)$. The second time interval is between $4 \mathrm{pm}$ and $6 \mathrm{pm}$, thus $T=6 \mathrm{pm}$ is the end of the business day.

Real values for both parties

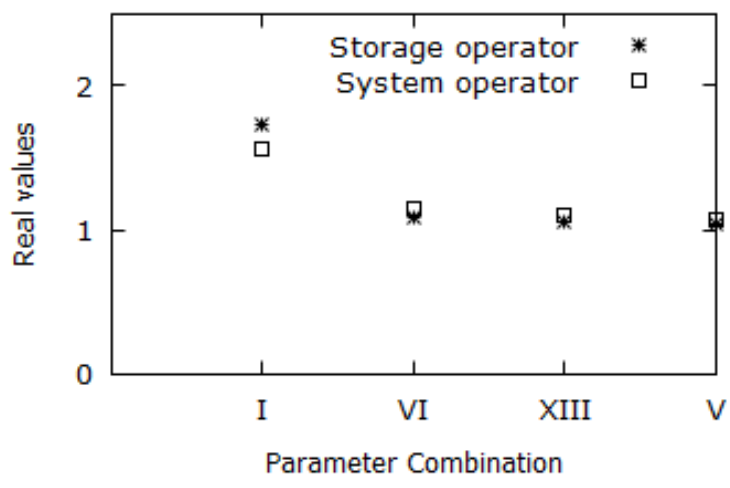

(a) Individual values
Real values for both parties

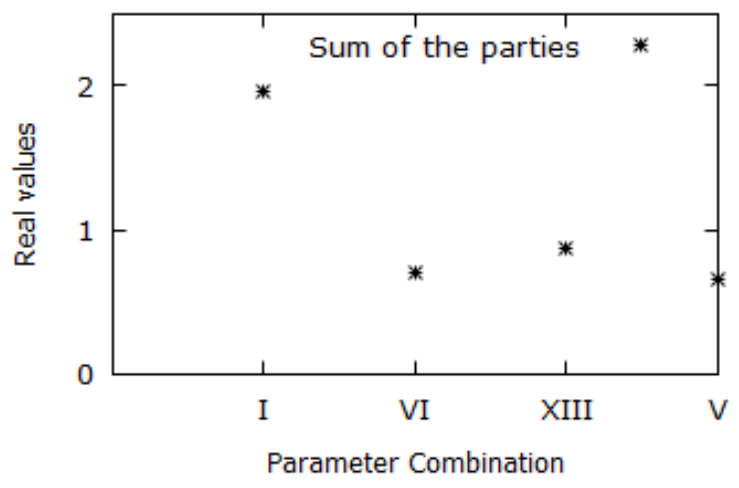

(b) Sum value

Figure 18: The ratio between corresponding real values that are received by dividing the values using actual $\mu=-94.7489$ with $\mu=0$ after applying 20000 daily cycles under Case (ii) where the second time interval is between $4 \mathrm{pm}$ and $6 \mathrm{pm}$, thus $T=6 \mathrm{pm}$ is the end of the business day. The corresponding real values are associated to different parameter combinations in Table 1, even though $C_{2}$ might be different but the rest coincide with that of the parameters in Table 1 The contract values denote the value in the beginning of the business day under 0 imbalance value and for initial case $(1, N)$. The second time interval is between $4 \mathrm{pm}$ and $6 \mathrm{pm}$, thus $T=6 \mathrm{pm}$ is the end of the business day. 
[1] Balancing Mechanism. https://www.elexon.co.uk/knowledgebase/what-is-the-balancingmechanism/. Accessed: 2017-01.

[2] Elexon Portal. https://www.elexonportal.co.uk/ Accessed: 2016-05.

[3] National Grid Balancing Services Incentive Scheme. http://www.nationalgrid.com/UK/Industryinformation/Electricity-system-operator-incentives/BSIS/. Accessed: 2016-03.

[4] René Aïd. A review of optimal investment rules in electricity generation. In Quantitative Energy Finance, pages 3-40. Springer, 2014.

[5] Michele Arnold and Göran Andersson. Model predictive control of energy storage including uncertain forecasts. In Power Systems Computation Conference (PSCC), Stockholm, Sweden, volume 23, pages 24-29, 2011.

[6] John P Barton and David G Infield. Energy storage and its use with intermittent renewable energy. IEEE transactions on energy conversion, 19(2):441-448, 2004.

[7] Trine Krogh Boomsma, Nigel Meade, and Stein-Erik Fleten. Renewable energy investments under different support schemes: A real options approach. European Journal of Operational Research, 220(1):225237,2012 .

[8] René Carmona and Michael Ludkovski. Valuation of energy storage: An optimal switching approach. Quantitative finance, 10(4):359-374, 2010.

[9] Alvaro Cartea and Marcelo G Figueroa. Pricing in electricity markets: a mean reverting jump diffusion model with seasonality. Applied Mathematical Finance, 12(4):313-335, 2005.

[10] Edgardo D Castronuovo and Joao A Pecas Lopes. Optimal operation and hydro storage sizing of a wind-hydro power plant. International Journal of Electrical Power \& Energy Systems, 26(10):771-778, 2004 .

[11] In-Koo Cho and Sean P Meyn. Efficiency and marginal cost pricing in dynamic competitive markets with friction. Theoretical Economics, 5(2):215-239, 2010.

[12] Sören Christensen. On the solution of general impulse control problems using superharmonic functions. Stochastic Processes and their Applications, 124(1):709-729, 2014.

[13] Kai Lai Chung and John B Walsh. Markov processes, Brownian motion, and Time Symmetry, volume 249 of Grundlehren der Mathematischen Wissenschaften. Springer, New York, NY, 2nd edition, 2005.

[14] Sara Eftekharnejad, Vijay Vittal, Gerald Thomas Heydt, Brian Keel, and Jeffrey Loehr. Impact of increased penetration of photovoltaic generation on power systems. IEEE transactions on power systems, 28(2):893-901, 2013.

[15] Nicolas Gast, Jean-Yves Le Boudec, Alexandre Proutière, and Dan-Cristian Tomozei. Impact of storage on the efficiency and prices in real-time electricity markets. In Proceedings of the fourth international conference on Future energy systems, pages 15-26. ACM, 2013.

[16] Reza Ghaffari and Bala Venkatesh. Options based reserve procurement strategy for wind generatorsusing binomial trees. IEEE Transactions on power systems, 28(2):1063-1072, 2013.

[17] Heiko Hahn, Silja Meyer-Nieberg, and Stefan Pickl. Electric load forecasting methods: Tools for decision making. European journal of operational research, 199(3):902-907, 2009.

[18] Lawrence E Jones. Renewable energy integration: practical management of variability, uncertainty, and flexibility in power grids. Academic Press, 2014. 
[19] Daniel S Kirschen and Goran Strbac. Fundamentals of power system economics. John Wiley \& Sons, 2004.

[20] Magnus Korpaas, Arne T Holen, and Ragne Hildrum. Operation and sizing of energy storage for wind power plants in a market system. International Journal of Electrical Power 85 Energy Systems, 25(8):599-606, 2003.

[21] Grigorios L Kyriakopoulos and Garyfallos Arabatzis. Electrical energy storage systems in electricity generation: Energy policies, innovative technologies, and regulatory regimes. Renewable and Sustainable Energy Reviews, 56:1044-1067, 2016.

[22] Xing Luo, Jihong Wang, Mark Dooner, and Jonathan Clarke. Overview of current development in electrical energy storage technologies and the application potential in power system operation. Applied energy, 137:511-536, 2015.

[23] John Moriarty and Jan Palczewski. Energy imbalance market call options and the valuation of storage. arXiv preprint arXiv:1610.05325, 2016.

[24] John Moriarty and Jan Palczewski. Real option valuation for reserve capacity. European Journal of Operational Research, 2016.

[25] MH Nehrir, Caisheng Wang, Kai Strunz, Hirohisa Aki, Rama Ramakumar, James Bing, Zhixhin Miao, and Ziyad Salameh. A review of hybrid renewable/alternative energy systems for electric power generation: Configurations, control, and applications. IEEE Transactions on Sustainable Energy, 2(4):392-403, 2011.

[26] Goran Peskir and Albert Shiryaev. Optimal stopping and free-boundary problems. Springer, 2006.

[27] Georg C Pflug and Nikola Broussev. Electricity swing options: Behavioral models and pricing. European journal of operational research, 197(3):1041-1050, 2009.

[28] Ramteen Sioshansi. Welfare impacts of electricity storage and the implications of ownership structure. The Energy Journal, pages 173-198, 2010.

[29] Gordon D Smith. Numerical solution of partial differential equations: finite difference methods. Oxford university press, 1985.

[30] Goran Strbac, Anser Shakoor, Mary Black, Danny Pudjianto, and Thomas Bopp. Impact of wind generation on the operation and development of the uk electricity systems. Electric Power Systems Research, 77(9):1214-1227, 2007.

[31] Dávid Zoltán Szabó and Randall Martyr. Real option valuation of a decremental regulation service provided by electricity storage. Phil. Trans. R. Soc. A, 375(2100):20160300, 2017.

[32] Matt Thompson, Matt Davison, and Henning Rasmussen. Natural gas storage valuation and optimization: A real options application. Naval Research Logistics (NRL), 56(3):226-238, 2009.

[33] John N Tsitsiklis and Yunjian Xu. Pricing of fluctuations in electricity markets. European Journal of Operational Research, 246(1):199-208, 2015.

[34] Yunjian Xu and Lang Tong. Optimal operation and economic value of energy storage at consumer locations. IEEE Transactions on Automatic Control, 62(2):792-807, 2017.

[35] Behnam Zakeri and Sanna Syri. Electrical energy storage systems: A comparative life cycle cost analysis. Renewable and Sustainable Energy Reviews, 42:569-596, 2015. 